\title{
On Joint Source-Channel Decoding and Interference Cancellation in CDMA-Based Large-Scale Wireless Sensor Networks
}

\author{
by \\ Chathura Illangakoon
}

\begin{abstract}
A Thesis
submitted to the Faculty of Graduate Studies of The University of Manitoba, in Partial Fulfilment of the Requirements for the degree of

Master of Science

in

Electrical and Computer Engineering
\end{abstract}

(C) by Chathura Illangakoon, June 21, 2013

Department of Electrical and Computer Engineering

University of Manitoba

Winnipeg, Manitoba R3T 5V6 Canada 


\section{Abstract}

Motivated by potential applications in wireless sensor networks, this thesis considers the problem of communicating a large number of correlated analog sources over a Gaussian multiple-access channel using non-orthogonal code-division multiple-access (CDMA). A joint source-channel decoder is presented which can exploit the inter-source correlation for interference reduction in the CDMA channel. This decoder uses a linear minimum mean square error (MMSE) multi-user detector (MUD) in tandem with a MMSE joint source decoder (JSD) for multiple sources to achieve a computational complexity that scales with the number of sources. The MUD and the JSD, then iteratively exchange extrinsic information to improve the interference cancellation. Experimental results show that, compared to a non-iterative decoder, the proposed iterative decoder is more robust against potential performance degradation due to correlated channel interference and offers better near far resistance. 


\section{Acknowledgements}

There are a number of people I wish to thank for making my experience as a masters student an interesting and rewarding period. First of all, I would like to thank my advisor Prof. Pradeepa Yahampath for his continual support and guidance in the last two years. His energy, patience, and judgment of students has been truly inspirational. In addition, his well-taught Topics in Signal Compression and Coding, and Information Theory courses boosted my enthusiasm towards information and communication theoretic research work. I wish to acknowledge the financial support from National Science and Research Council (NSERC).

Secondly, I would like to thank all the professors in our department and outside the department for their invaluable support and guidance. Also I would like to thank the administrative staff of the Faculty of Graduate Studies and the department of Electrical and Computer Engineering.

My sincere gratitude goes to all my colleagues in the CNER lab whose support and friendship was invaluable. Also I have to thank all my friends, in and outside of University of Manitoba, for making the years so enjoyable.

Finally, I would like to thank my parents for inspiring a love of learning and giving me the freedom to follow my passion in math and science. I can't imagine coming so far without their love and dedication. 


\section{Contents}

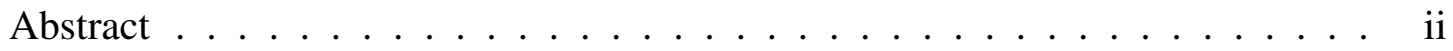

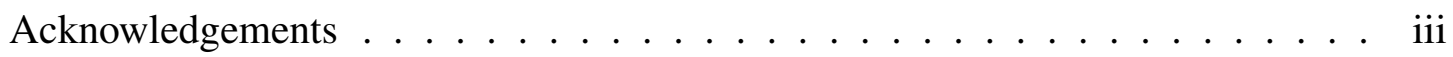

List of Tables . . . . . . . . . . . . . . . . . . . . vi

List of Figures . . . . . . . . . . . . . . . . vii

1 Introduction 1

1.1 Background and Motivation . . . . . . . . . . . . . . 1

1.2 The issue of computational complexity in decoding . . . . . . . . . . . . 4

1.3 The effect of non-orthogonal MAC for correlated sources ........................ 5

1.4 Summary of Contributions . . . . . . . . . . . . . . . . . 7

1.5 The outline of the thesis . . . . . . . . . . . . 7

2 Background $\quad 8$

2.1 Source coding . . . . . . . . . . . . . . . . . 8

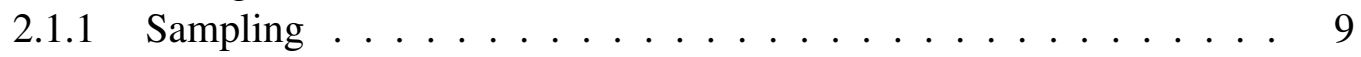

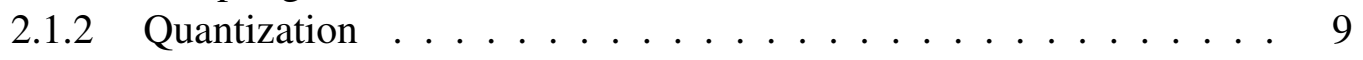

2.1.3 Soft VQ decoding . . . . . . . . . . . . . . . . 14

2.2 Multi-user communication . . . . . . . . . . . . . 15

2.2.1 Random Multi-access . . . . . . . . . . . . . . . . . . . 17

2.2 .2 CDMA ....................... 17

2.3 Multi-user detection . . . . . . . . . . . . . . . . 20

2.3.1 The optimal multi-user detector ............. 21

3 Iterative Joint Source Channel Decoder 23

3.1 Problem description . . . . . . . . . . . . . . . . . . . . . 24

3.2 The MMSE decoder . . . . . . . . . . . . . . . . . 25

3.3 The proposed Iterative JSC decoder . . . . . . . . . . . . . . . 26

3.3.1 A soft MUD with interference cancellation . . . . . . . . . . 28

3.3.2 Optimal JVQ decoder . . . . . . . . . . . . . . . 32

4 Simulation Results and Discussion $\quad 38$

4.1 Performance comparison between iterative and non-iterative JSC decoding 39

4.2 Near-far resistance of the proposed JSC decoder . . . . . . . . . . . . . 44

4.3 Performance comparison of low-complexity JSC decoders . . . . . . . . 46 
4.4 Performance of the proposed decoder on different

CDMA channels . . . . . . . . . . . . . . . . . . . . . 49

5 Conclusions and Future Work $\quad 54$

References ....................... 57 


\section{List of Tables}

4.1 The RSNR improvement of MMSE MUD + JVQ decoder through iterations. The CDMA cross-correlation matrix is $\mathbf{R}_{1}$. . . . . . . . . . . . . 40

4.2 The RSNR improvement of optimum MUD + JVQ decoder through iterations. The CDMA cross-correlation matrix is $\mathbf{R}_{1}$. . . . . . . . . . . . . 40

4.3 The RSNR improvement of MMSE MUD + JVQ decoder through iterations. The CDMA cross-correlation matrix is $\mathbf{R}_{2}$. . . . . . . . . . . . 40

4.4 The RSNR improvement of optimum MUD + JVQ decoder through iterations. The CDMA cross-correlation matrix is $\mathbf{R}_{2}$. . . . . . . . . . . . . 42 


\section{List of Figures}

1.1 Block diagram of a WSN over a multi-access channel. . . . . . . . . . 2

1.2 Block diagram of a non-orthogonal MAC for two users. . . . . . . . . . 5

2.1 Decision points and output levels of an N-point scalar quantizer. . . . . . 10

2.2 Flow chart of the Lloyd algorithm for quantizer design. . . . . . . . . . 13

2.3 Two-dimensional quantization with hexagonal partition. . . . . . . . . . . 14

2.4 A communication system. . . . . . . . . . . . . . . . . 15

2.5 Basic structure of the multi-access communication with four users. . . . . 16

2.6 A simple CDMA model with two users. . . . . . . . . . . . . . . 17

2.7 Orthogonal signature waveforms assigned to each user. . . . . . . . . 18

2.8 Demodulation of transmitted bit streams using correlation detectors. $r(t)$ is the channel output signal. . . . . . . . . . . . . . . . . . . 19

2.9 Conventional correlation multi-user detector. . . . . . . . . . . . 20

3.1 The WSN model considered in this thesis. . . . . . . . . . . . . . . 24

3.2 Block diagram of the proposed iterative JSC decoder for correlated sources. 27

3.3 The equivalent AWGN channel. . . . . . . . . . . . . . . . . 30

3.4 The factor-graph representation of joint pdf in (3.27) . . . . . . . . . . 33

3.5 The factor-graph representation of $\prod_{k=1}^{K}\left(\prod_{n=0}^{L-1} \phi_{n}\left(i_{k}\right)\right) P\left(I_{k}=i_{k} \mid I_{k-1}=\right.$

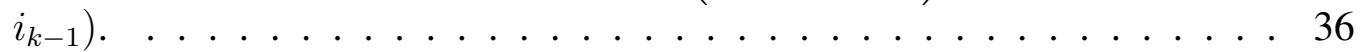

4.1 The linear array of sensors under consideration. . . . . . . . . . . 38

4.2 Performance comparison between iterative and non-iterative JSC decoding of 4 correlated Gaussian sources over a CDMA channel with destructive interference.

4.3 Performance comparison between iterative and non-iterative JSC decoding of 4 correlated Gaussian sources over a CDMA channel with constructive interference. . . . . . . . . . . . . . . . . . 43

4.4 Near-far performance (User-1 is illustrated) of the proposed JSC decoder in a four-user system with the CDMA cross-correlation matrix $\mathbf{R}_{1}$. All users employ scalar quantizers. The power of user one is a constant given by $C S N R_{1}=10 \mathrm{~dB}$. The interfering users have equal powers given by $C S N R_{k}-C S N R_{1}(\mathrm{~dB}), k=2,3,4 \ldots \ldots \ldots \ldots$

4.5 Near-far performance of the proposed JSC decoder in a four-user system with the CDMA cross-correlation matrix $\mathbf{R}_{2} \ldots \ldots \ldots \ldots \ldots$ 
4.6 A comparison between low-complexity JSC decoders in a four-user system with the CDMA cross-correlation matrix $\mathbf{R}_{1}$. All users employ scalar quantizers

4.7 A comparison between low-complexity JSC decoders in a four-user system with the CDMA cross-correlation matrix $\mathbf{R}_{2} \ldots \ldots \ldots \ldots$. . . . . 48

4.8 Performance of the proposed decoder in three different CDMA channels. The corresponding cross-correlation matrices are given by $\mathbf{R}_{1}, \mathbf{R}_{2}$ and $\mathbf{R}_{3}$. . 50

4.9 Performance of the proposed decoder in an eight user system with different CDMA channels. . . . . . . . . . . . . . . . . . . 52 


\section{Chapter 1}

\section{Introduction}

In recent years, the research on wireless sensor networks (WSN) has been extensive and revolutionizing, promising a significant impact on the society through a vast range of possible applications. The MIT Technology Review ranked large-scale, low-power, economical WSNs $[1,2]$ as the number one emerging technology. WSNs are usually mission oriented and application specific, for example, monitoring physical or environmental conditions, such as temperature, sound, pressure, etc. Thus, the sensors in a WSN usually have to operate under a set of unique constraints, presenting new and difficult challenges to the researchers. In this thesis, as may be the case in many practical scenarios, we consider a WSN with large number of sensors observing correlated sources, connected to a central location (e.g., base station) through a shared communication channel. The block diagram of a WSN model with $K$ sensors is illustrated in Figure 1.1.

\subsection{Background and Motivation}

Consider the system shown in Figure 1.1, At the transmitter-end, the sensor observation $X_{k}$ of sensor $k$ is first quantized, and the quantizer output $I_{k}$ is binary encoded to generate a bit stream $\mathbf{b}_{k}$ (source-data). This process is called source encoding. In an effective communication system the encoded bit stream is expected to be transmitted to the receiver-end with 


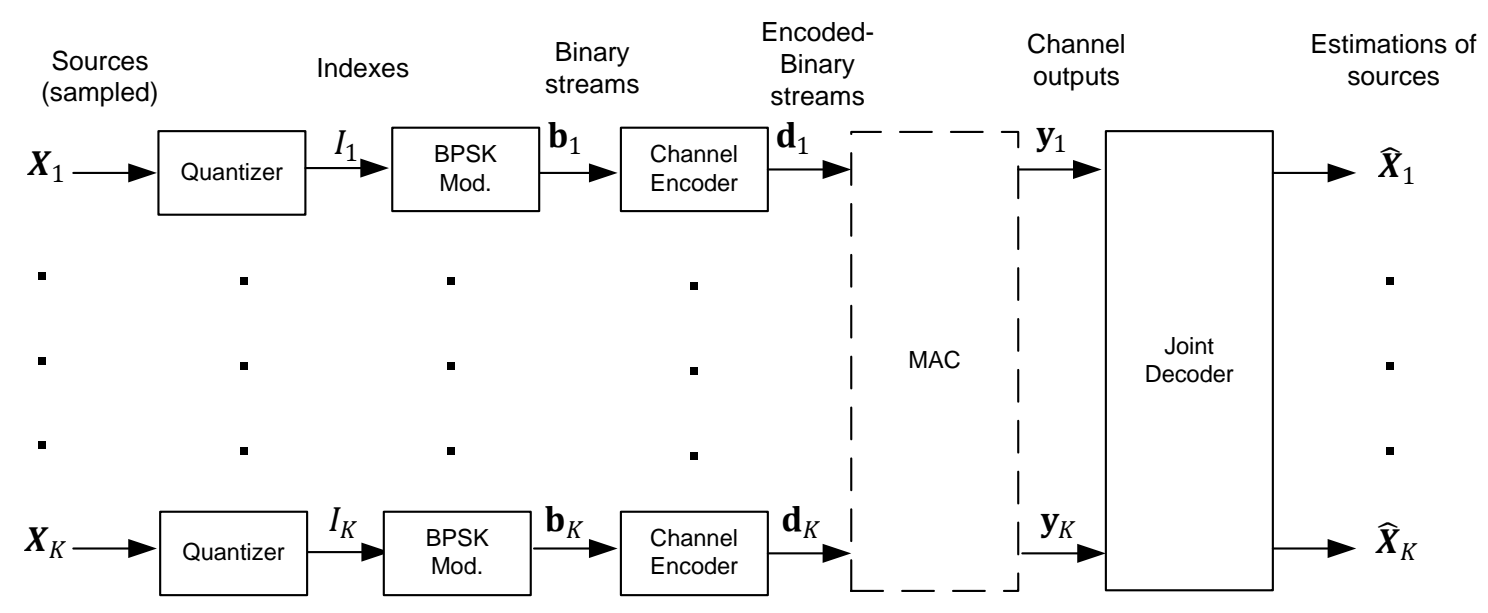

Figure 1.1: Block diagram of a WSN over a multi-access channel.

minimum distortion, preserving as much information as possible. The channel coding is used to protect source-data against the channel imperfections, by introducing redundancy to the transmitted bit stream. In the Figure 1.1, even though the channel coding block is indicated as a separate block from the source encoding, source and channel coding have to be jointly implemented to achieve an optimal performance in a multiuser communication system [3] with correlated sources. As such in this thesis, we consider a joint source-channel (JSC) coding scheme, in which the redundancy across users due to the inter-source correlation is exploited to mitigate the imperfection in the multiple access channel (MAC). Such a coding system in which both source coding and channel coding is achieved by a single code is referred to as JSC code. The encoded signal is then transmitted to the central location via the MAC. In the central location, a joint decoder will reconstruct the sensor observations. Our understanding of WSN reveals that, in many practical scenarios, there exist a large amount of correlation among observations produced by closely-placed sensors. Such correlation can be exploited in a joint decoder, in which the decoding of one user is carried out by considering signals transmitted by all users.

In medium to large WSNs, sharing of the communication medium becomes a critical issue. A multiuser communication channel may consist of many independent or dependent parallel channels, with one channel for each user. Two classical channel sharing techniques 
were developed based on frequency devision multiplexing and time devision multiplexing, known as frequency devision multiple access (FDMA) and time devision multiple access (TDMA). With large enough frequency or time guard intervals FDMA and TDMA channels can be assumed to be independent, that is there will be no interference among parallel channels [4]. In a WSN, in which the number of users may vary with time, static channel sharing schemes such as TDMA and FDMA can be ineffective, as far as the channel allocation is concerned (see [5, Ch. 1]. Therefore, one of the more practical channel sharing scheme in this case is code division multiple access (CDMA), in which the channels are allocated dynamically, by assigning a unique signature waveform for each user (see [5, Ch. 2]. The cross-correlation among CDMA signatures assigned to two users will determine if the two channels are independent or dependent, i.e., if there is mutual interference among the two users. In many previous studies, JSC decoding of correlated sources over a interference free channels (i.e., orthogonal channels) have been considered [6-9]. In CDMA, the most commonly employed signature waveform structure is known as directsequence spread spectrum. In direct-sequence CDMA (DS-CDMA), if the number of users are greater than the number of chips per symbol, the orthogonality among parallel channels is not practical [10]. This is the case in large-scale WSN. One might easily conceive the idea that mutual interference (non-orthogonality in the parallel channels) among the users always degrades the performance of a multiuser communication system. This is in fact not true. While an orthogonal MAC is optimal for transmitting independent sources, orthogonal CDMA, in general, is not optimal for transmitting correlated sources in a multiuser communication system [11]. In this thesis, our focus is twofold. First to investigate the complexity issue associated with the joint decoding of a large WSN and propose a good low-complex JSC decoding solution. The second issue we focus on is the effect of a particular set of CDMA signatures on the performance of a multi-user communication system used in a WSN. 


\subsection{The issue of computational complexity in decoding}

In this thesis, the mean square error (MSE) is used as the performance measuring criterion. In a similar communication model as illustrated in Figure 1.1, the minimum MSE (MMSE) joint source-channel decoding over non-orthogonal CDMA channel has been previously considered in [12]. However, the MMSE decoder considered in [12] has a computational complexity that is exponential in the number of sources $O\left(2^{K}\right)$, which is prohibitive for WSNs with medium to large number of users. Low complexity joint decoders for large scale WSNs with orthogonal CDMA channels have been previously proposed in $[8,9]$. Such decoders can be used on non-orthogonal channel as well, if in tandem a multi-user detector (MUD) is used. A MUD will account for multiuser interference due to the nonorthogonality in the MAC and will present orthogonal channels to the joint decoders [5]. However, such an approach can be viewed as a case of separate source-channel decoding. Hence, It is impractical to use inter-source correlation to mitigate multi-user interference in a MUD and therefore we lose the redundant information that can be used for errorprotection. In contrast to previous work, in this thesis we present and investigate a low complexity iterative JSC decoder for correlated sources and a CDMA channel, which exploits inter-source correlation for improved interference cancellation. More specifically, we extend the joint vector quantization (VQ) decoder presented in [9], to obtain a low complexity JSC decoder wherein the key idea is to perform iterative decoding, by using the joint VQ decoder in tandem with a soft-input soft-output MUD. With soft outputs obtained from a MUD, the joint VQ decoder utilizes all of the received information. As a result the joint VQ decoder has an infinite output alphabet. Such soft VQ decoding was studied for the additive white Gaussian noise channel in $[13,14]$. The overall computational complexity

of the iterative decoder proposed in this thesis is $O\left(K^{2}\right)$ for $K>>1$, making the decoding computationally tractable for large-scale WSNs. 


\subsection{The effect of non-orthogonal MAC for correlated}

\section{sources}

In this thesis, the impact of CDMA signatures used with correlated sources is also investigated. As mentioned earlier an orthogonal MAC is not necessarily optimum for correlated sources. Even though we do not derive an analytical method to determine the optimum or a near optimum set of signatures, a heuristic approach based on intuitive knowledge is taken to investigate the effect of CDMA signatures using computer simulations. These simulation results clearly show the significance of choosing the right set of CDMA signatures for correlated sources. In order to demonstrate that the channel interference in a MAC does not always degrade the performance of decoding of correlated sources, consider the following example.

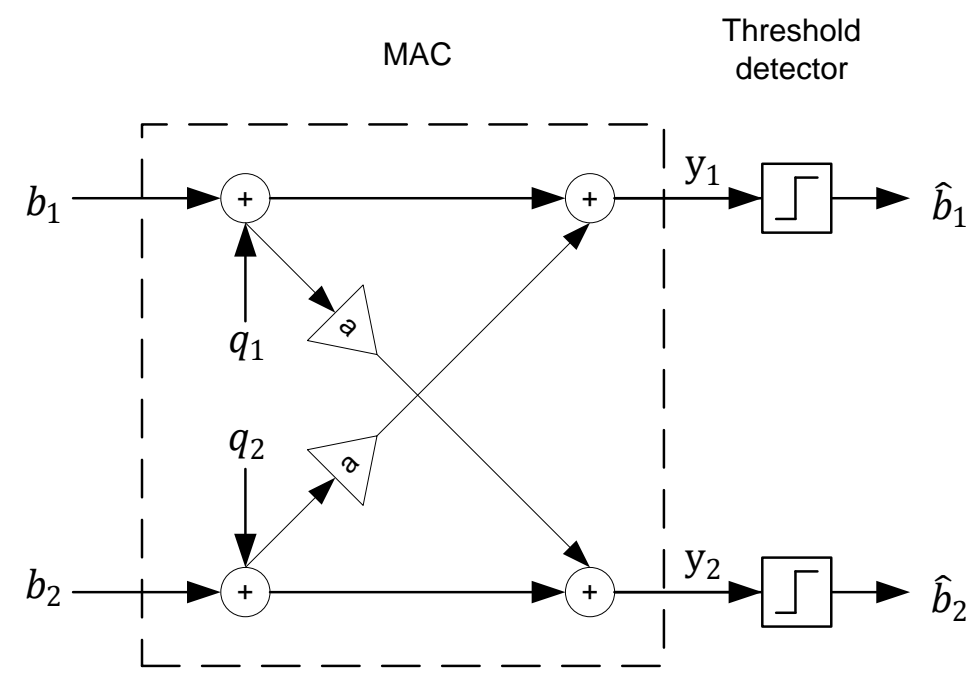

Figure 1.2: Block diagram of a non-orthogonal MAC for two users.

In Figure 1.2, $b_{1}$ and $b_{2}$ are two binary sources $\left(b_{i} \in\{+1,-1\}\right.$, i.e., the baseband equivalent of binary phase shift keying (BPSK)), and the constant $a(|a|<1)$ is a known parameter, which determines the level of interference between two parallel channels. In Figure $1.2 q_{1}$ and $q_{2}$ represent additive noise in the parallel channels. In this model, if $a=0$, clearly the parallel channels become interference free, i.e. orthogonal channels. 
However, if $a$ is non-zero, two parallel channels are not independent anymore. As we are considering correlated sources, most of the time, we are likely to have $b_{1}=b_{2}$. In such cases, the outputs $y_{i}, i=1,2$ of the two parallel channels are given by

$$
\begin{aligned}
& y_{1}=(1+a) b+q_{1}+a q_{2}, \\
& y_{2}=(1+a) b+q_{2}+a q_{1},
\end{aligned}
$$

where $b_{1}=b_{2}=b$. As one can see, if $a$ tends to $-1, y_{i}$ is dominated by noise component at the channel output, and therefore estimates $\hat{b}_{i}$ obtained using a detection unit is not an accurate approximation of $b_{i}$. However, if $a$ tends to $+1, y_{i}$ is dominated by the channel input signal, and hence the estimate is much more reliable. Also notice that, compared to the case when $a=0$ (i.e. independent channels), when $a \rightarrow 1$, the input signal is much more dominant in the channel outputs. The above example shows that the interference in a MAC can improve the fidelity of decoding for correlated sources. When channel interference among set of correlated users lead to an increase in the strength of the decoder input signals of the users, we refer to it as constructive interference. On the other hand, destructive interference occur when the signal strength at the decoder input degrades as a result of channel interference among correlated users. Since in a large WSN, the sensor locations may not be fixed or known, the iterative decoder proposed in this thesis is designed to be robust against constructive or destructive interference in the channel. This robustness is achieved by using bit-level interleavers, and hence making the channel inputs of different users statistically independent. However, compared to a non-iterative (non-interleaved) decoder, which can be optimized if CDMA are chosen to match the sources, it is difficult to optimize the proposed decoder by matching the CDMA signatures to the sources. Therefore we also discuss the possibility of selecting a set of CDMA signatures optimal for a non-iterative decoding of correlated sources. 


\subsection{Summary of Contributions}

In this thesis, we present an iterative approach to JSC decoding of correlated sources over a DS-CDMA channel. The methodologies adopted to address the complexity issue in a decoding of a large WSN is extensively discussed. We demonstrate that the proposed JSC decoder is robust against the (possible) destructive interference produced by the multi-user channel. A numerical analysis on the optimization of CDMA signatures for correlated sources, based on a heuristic approach, is also presented in this thesis, while leaving theoretical analysis on that problem as a future work.

\subsection{The outline of the thesis}

The rest of the thesis is organized as follows. In Chapter 2 we begin with briefly explaining fundamental concepts and background studies that are relevant to the development of this thesis. Chapter 3 presents the derivation of the proposed iterative JSC decoder, focusing on reducing the computational complexity of joint decoding of a large number of correlated sources. The numerical results and a discussion of the implications of the results are presented in Chapter 4. Chapter 5 concludes the thesis with a discussion of possible future work. 


\section{Chapter 2}

\section{Background}

\subsection{Source coding}

Shannon in his 1948 paper [15] wrote "the fundamental problem of communication is that of reproducing at one point either exactly or approximately a message selected at another point". This problem has been separated into few sub problems for effective analysis. In communication, source coding is the conversion of a signal into an efficient digital representation, while channel coding is a technique used to correct errors of a digital transmission over a noisy channel. In Shannon's development of information theory, he has shown that a nearly optimal communication system is possible by designing an optimal source code for the source and an optimal channel code for channel separately. With that knowledge it is worthy to look at the source coding as a separate issue in a communication system. However the positive coding theorems [3] in information theory shows such optimal separability is only possible with codewords of infinite length [15], which causes an infinite delay. On the other hand, there are channels for which the separation theorem is not valid. Multiuser channels are one such class of channels. Furthermore as it was implied earlier, when the delay is a limiting factor, the source-channel separability is not possible without losing the overall optimality. In these cases, the optimal solution is joint sourcechannel coding. In the following section, source coding is discussed in order to relate its 
importance to this thesis. In Sec. 2.2, multi-user communication is discussed.

\subsubsection{Sampling}

The natural sources of signals (information) such as temperature, pressure, or any other environmental measurement are analog and continuous in time. The starting step of converting such natural waveforms in to a digital form is sampling. The well known sampling theorem [16] shows that an analog waveform with finite bandwidth can be represented by countable set of points in time without losing any information. This indeed is a consequence of the fact that signal is band-limited. The intuitive explanation is that, if the signal is band-limited, then the signal will not fluctuate too quickly and hence the true signal values between two sampling points should follow a smooth curve. To recover the complete waveform, a process of interpolation or smoothing can be used. In smoothing, a smooth curve is constructed which passes through all sample values of the signal. However the solution to be unique, the sampling rate must be at least twice the bandwidth of the signal (Nyquist-Shannon sampling theorem [16]).

\subsubsection{Quantization}

Quantization is one of the most important steps in source coding. A quantizer observes a random variable and finds an approximate value according to a pre-defined rule. An input to a quantizer is usually a sample from an analog waveform and the output is digital (specified by a finite set). The input to the quantizer can either be a scalar (scalar quantization) or a vector (vector quantization).

\section{Scalar quantization}

A quantizer can be viewed as two successive operations of mapping. Two operations are identified as encoding (digital representation of the input signal) and decoding (estimation

of the original signal). In scalar quantization, input to the encoder is a real number (i.e. a 
scalar). The encoder maps the input into an integer. The decoder maps the integer into a real number from a finite set. Figure 2.1 illustrates an $N$-point scalar quantizer ( $N$ number of output levels) with its defining points. The values $x_{i}, i=1, \ldots, N$ are often called the boundary points or decision points. The encoder is defined by set of regions $R_{i}$ formed by intervals between successive end points. Each region $R_{i}$ (or a cell) can be labeled by an integer. An input which falls in to a particular cell is mapped to the assigned integer. The decoder maps the encoder output in to corresponding output level $y_{i}$. Importance of separating quantization in to two processes is that, in a communication system encoding takes place at the transmitter while decoding is done at the receiver.

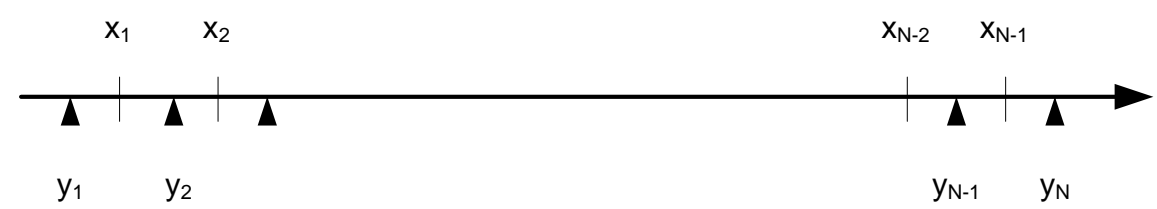

Figure 2.1: Decision points and output levels of an N-point scalar quantizer.

\section{Implementation of a scalar quantizer}

The objective of quantization is to provide a finite-precision description to its input value. However in the design process, the input is an unknown quantity. In this case one can model the input as a random variable, given its statistical description (probability density function). As a result, the error introduced during the quantization process can be modeled as a random variable. Having modeled the quantization error, the overall (average) quality degradation due to the approximation can be computed as a statistical average of the error. In almost all practical cases, the mean square error (MSE) is used as the average distortion measure. With the MSE as the distortion measure for a given input signal, $X$, and quantizer $Q=\left\{y_{i}, R_{i} ; i=1,2, \ldots, N\right\}$, distortion $D$ is given by

$$
D=E\left[(X-Q(X))^{2}\right]=\sum_{i=1}^{N} \int_{R_{i}}\left(x-y_{i}\right)^{2} f_{X}(x) d x
$$


where $f_{X}(x)$ is the probability density function (pdf) of the random variable.

\section{Uniform quantizer}

The most common implementation of a quantizer is the uniform quantizer. Uniform quantization has been mostly used for general-purpose analog-to-digital conversion. A uniform quantizer is a regular quantizer [17], which follows the following properties: (i) the decision points are equally spaced, and (ii) the output level of the bounded regions are the mid points, of the quantization regions. With the uniform quantization, provided that the $N$ is large enough (high resolution quantization), the overall distortion $\mathrm{D}$ is given by

$$
D=\frac{\Delta^{2}}{12}
$$

where $\Delta$ is the quantization interval.

In the case of high resolution quantization, the performance of a uniform quantizer only depends on quantization interval $\Delta$ and is independent of the input pdf. The quantizer which is optimized for the input, for a fixed number of output levels is discussed in the next section.

\section{Optimal quantizer}

The primary objective of a quantizer design is to achieve minimum distortion by determining the optimal reproduction levels and the partition regions. The design problem in more explicit terms is that for a given distortion measure (e.g., MSE), to find reproduction points $y_{i}$ and partition cells $\left\{R_{i}\right\}$ that minimize $D$ given by (2.2).

In general, it is difficult to find a closed-form solution to the aforementioned problem. In [17], the design process is simplified by considering two separate problems based on the necessary conditions for the optimal design. The two problems are,

1. The optimal encoder for a given decoder:

In more explicit terms, this is a task of finding the best partition (which minimizes 
the distortion) for a given set of reproduction values (codebook). The condition for the optimality as proved in [17] is the nearest neighbor condition which requires that, a quantization region should consist of all input values closer to its reproduction value than to any other reproduction value. This requirement is easily achieved by choosing decision points to be the midpoint between two adjacent output levels, i.e.

$$
x_{i-1}=\frac{\left(y_{i-1}+y_{i}\right)}{2} \text {. }
$$

The nearest neighbor rule fully defines the partition of the quantizer.

2. The optimal decoder for a given encoder:

The problem at hand is finding the optimal codebook for a fixed partition. This in fact is a one necessary condition for an optimal quantizer (i.e. the codebook must be optimal for the given partition). The centroid condition is both the sufficient and necessary condition for optimality, provided the MSE used as the distortion measure [17]. Given a partition, the optimal codebook for a given random variable (i.e. the pdf of the input signal) is given by

$$
y_{i}=\int_{R_{i}} x f_{X \mid R_{i}}(x) d x=\frac{\int_{R_{i}} x f_{X}(x) d x}{\int_{R_{i}} f_{X}(x) d x} .
$$

In [18], (2.3) and (2.4) are used iteratively, until the average distortion converges to a stationary point. Figure 2.2 illustrates the basic design algorithm presented in [18].

\section{Vector quantization}

Vector quantization (VQ) is the generalization of scalar quantization to the quantization of vectors. Many of the design techniques used for scalar quantization can be generalized to deal with the vectors. A vector quantizer $\mathrm{Q}$ of dimension $k$ and size $N$ is a mapping from a vector in $k$-dimensional Euclidean space into a finite set containing $N$ reproduction points. In [17], it has been shown that VQ is the optimal solution to the quantization problem as $k \rightarrow \infty$. The two potential advantageous of VQ over scalar quantization are: 


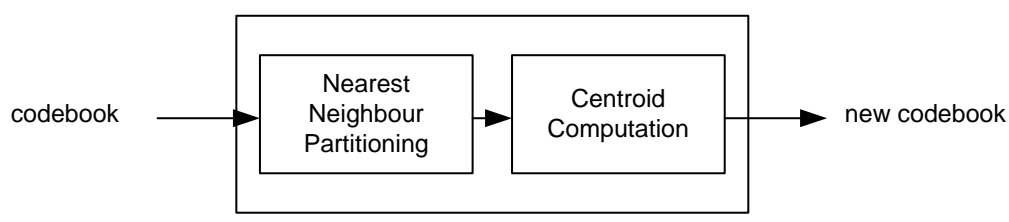

Lloyd iteration for codebook improvement

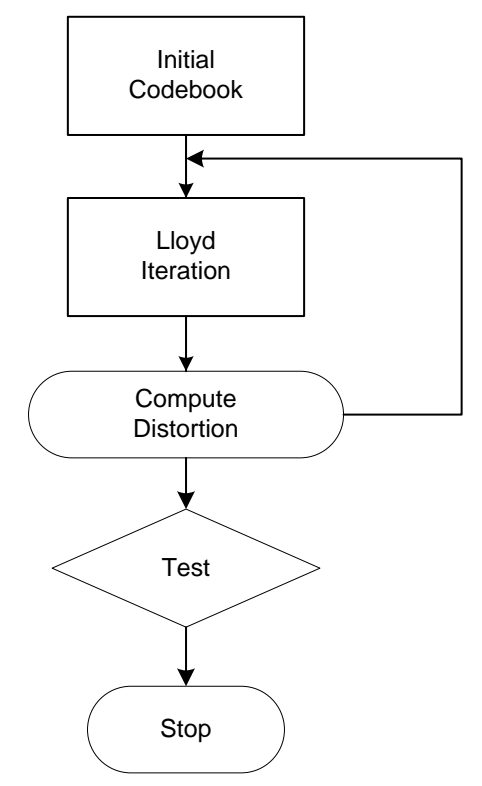

Figure 2.2: Flow chart of the Lloyd algorithm for quantizer design.

1. The ability to exploit statistical dependency between the vector elements

2. The additional freedom of forming differently shaped quantizer cells in a high dimensional space

When a sequence of random variables (a sampled waveform) is quantized, improved performance can be obtained, compared to scalar quantization, by partitioning data in to vectors and applying VQ to each vector. And also if the number of reconstruction points per each data sample is fixed, the performance of quantization process can be improved by increasing the dimensionality of the VQ. In other words, long term statistical dependency among the signal samples can be exploited (also the additional freedom to form different quantizer cell shapes in the higher dimensional vector space). The increase of dimension of dimension $k$ in VQ brings an additional complexity to the quantization process (note that the nearest neighbor search of codewords is an exhaustive process). In [17], it is shown that 
the complexity of VQ grows exponentially with the dimension $k$. In a situation where the encoder is constrained by its limited computational power, implementation of VQ may be impractical. Therefore, in such situations, scalar quantization is more practical than VQ. A detailed discussion on the design of VQ can be found in [17].

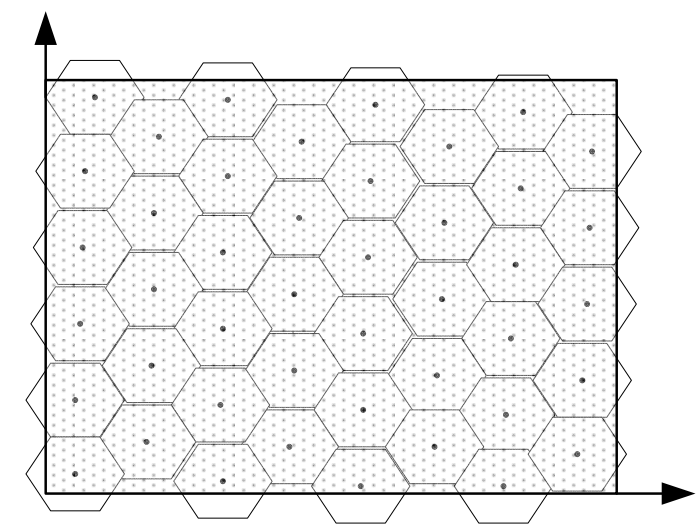

Figure 2.3: Two-dimensional quantization with hexagonal partition.

\subsubsection{Soft VQ decoding}

A conventional VQ decoder is defined by a lookup table of finite size $N$. This is also referred to as hard decoding. However, when the decoder has access to continues-valued channel outputs, the decoder can perform optimal estimation of the quantizer input. This approach is known as soft decoding. The first approach to soft decoding for VQ over a noisy channel was proposed in [13]. In contrast to hard decoding, a soft VQ decoder does not implement prior thresholding, instead it uses all soft (un-quantized) channel outputs. Numerical simulations confirm that soft decoding outperforms hard decoding [12].

\section{Optimum soft decoder}

A simple point-to-point communication system is illustrated in Figure 2.4. From estimation theory, the optimum decoder for the squared-error distortion measure, computes the conditional expectation of the source vector based on the channel output $y$ [13]. Therefore 


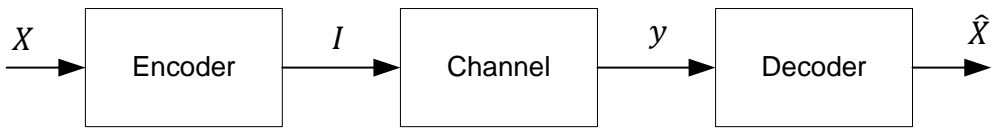

Figure 2.4: A communication system.

the optimal decoder output is given by

$$
\hat{X}=E\{X \mid y\} .
$$

By conditioning on the event that $i$ is transmitted and using the fact that $X$ is independent of $R$ given $I, \hat{X}$ can be expressed as,

$$
\hat{X}=\sum_{i=1}^{N} E\{X \mid i\} P(I=i \mid y),
$$

where $E\{X \mid i\}$ is the centroid of the $i$ th quantization cell, and $P(i \mid y)$ is the probability of $i$ is transmitted, given the channel output $y$. The decoder utilizes all of the received soft information $y$, in effect, has an infinite output alphabet.

\subsection{Multi-user communication}

As communication systems have grown from classical point-to-point communication to all-connected networks, the studies and analysis of multi-user communication have become important. In multi-access communication, the goal is to ensure the reliable data transmission while using the transmission medium efficiently by multiple users. In the communication model to be discussed in this thesis multiple users are connected to a receiver through a shared channel, a WSN is an example for such a system. In multi-user communication, the receiver obtains a superimposition of signals transmitted by different users. With the additive noise in the channel, the received signal is a noisy version of the superimposed signal. The separation of superimposed data polluted by channel noise is the fundamental problem of the multi-user communication.

The channel shown in Figure 2.5 is referred to as a multiple access channel (MAC). 


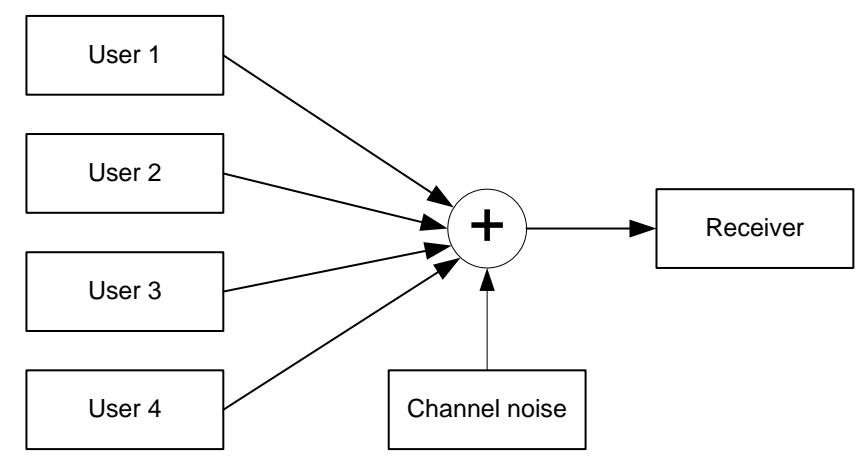

Figure 2.5: Basic structure of the multi-access communication with four users.

Two classical MACs, frequency-division multiple access (FDMA) and time-division multiple access (TDMA) were invented a few decades ago. The introduction of frequency modulation enabled several signals to coexist in the space at the same time without mutual interference (if the each signal is band-limited and the carrier frequency gaps are large enough) by using different carrier frequencies. In FDMA each user is assigned a different carrier frequency. At the receiver-end, each user signal is demodulated and filtered out. In TDMA, underlying technology is time-division multiplexing. In TDMA the time-line is divided into separate slots, and each slot will be assigned to a different user in a round robin manner. Unlike in FDMA, the user signals need to be digitized prior to multiplexing. On the other hand, for a reliable transmission, digital data streams need to be synchronized in time. With geographically separated users the synchronization can be difficult (e.g., in a WSN, sensors can be randomly located in space, and hence sharing a common clock may be impractical). In comparison, it can be noted that FDMA allows users to access the channel asynchronously in time, whereas in TDMA all the users must be synchronized to a single clock. However in FDMA each user is assigned a fixed bandwidth for its transmission. In such a static system, the addition or a departure of a user will have to be dealt with additional complexity to maintain the effectiveness and reliability of the system. It is important to note that WSNs are dynamic, and many sensors may join or depart from the network during its operation. The static channel sharing can be problematic in such situations. Therefore allocating resources dynamically still remains a problem in FDMA 
as well.

\subsubsection{Random Multi-access}

Random multi-access communication is a diversion from the static channel sharing discussed earlier. A user is allowed to access the transmission medium whenever it requires. In this approach, a user transmits as if it were the only user of the channel. Since the users are not coordinated, the possibility of inter-user interference is inevitable. If the transmitted signal cannot be demodulated at the receiver, as a result of a simultaneous transmission of multiple users, the users will have to be notified and the collided messages have to be re-transmitted (re-transmission techniques are discussed in [5]). It is realized that in a communication network where a number of users transmit continuously, a random multi-access scheme is not the most appropriate for a MAC. The techniques discussed so far do not facilitate more than one transmitter to occupy a given time-frequency slot. For a large dynamic network such as a WSN, a reliable MAC with dynamic channel allocation is required.

\subsubsection{CDMA}

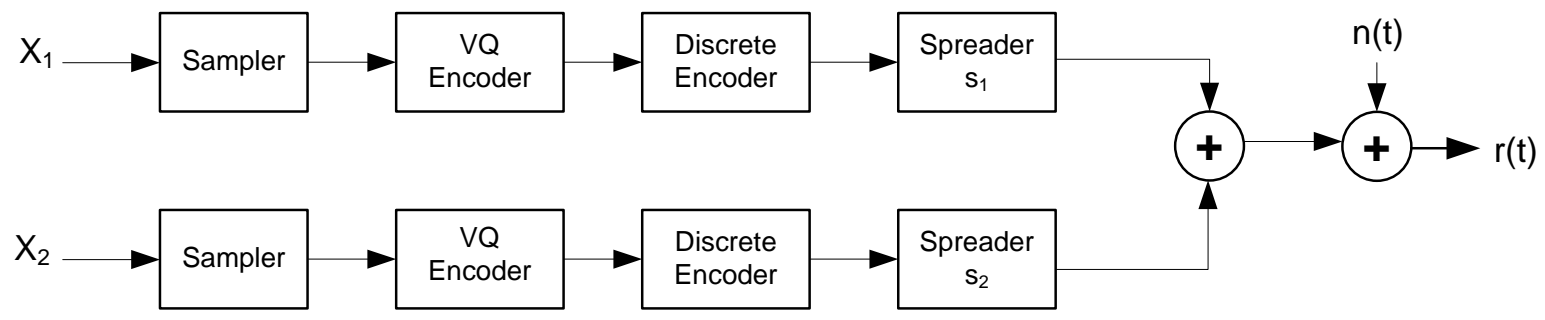

Figure 2.6: A simple CDMA model with two users.

Figure 2.6 illustrates how code division multiple-access (CDMA) resolves the multiuser communication problem. $X_{1}$ and $X_{2}$ are two sources (i.e. two analog waveforms) and as explained in the previous section, VQ encoders preceded by samplers produce two equivalent discrete sources of $X_{1}$ and $X_{2}$ respectively. The binary encoder generates a sequence 
of bits for each discrete-valued (index) it receives. In CDMA, channel allocation or sharing is implemented by assigning a unique waveform, called a signature waveform, to each user.

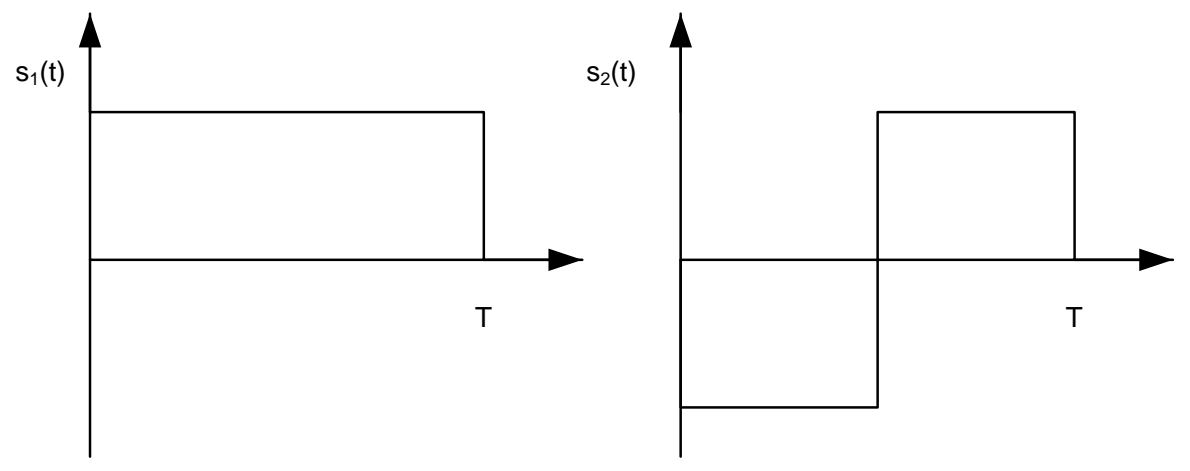

Figure 2.7: Orthogonal signature waveforms assigned to each user.

An example of orthogonal waveforms is shown in Figure 2.7. Both signals overlap in the time and frequency domains. However the inner product of the two signals is zero (i.e. the two signals are orthogonal). The inner product between two time domain signals is defined as the cross correlation between the two signals at zero time lag, given by

$$
<s_{1}, s_{2}>=\int_{T} s_{1}(t) s_{2}(t) d x
$$

In this two-user CDMA communication system, user1 and user2 are antipodally modulated using the signals $s_{1}$ and $s_{2}$, respectively. Assuming that transmission rates of both the users are equal and that the bit epochs are perfectly aligned, the demodulation of the bit streams can be easily done, despite the fact that the two waveforms overlap in both frequency and time. The receiver will observe a noisy version of superimposed signals. Given that the additive noise is white and Gaussian, correlation detector illustrated in Figure 2.8 is optimal (i.e., minimizes the bit-error-rate [5]) for demodulation.

With the assumption of a synchronous system and orthogonal signature waveforms $s_{1}$ and $s_{2}$, the detected bit stream of user1/user2 is not affected by the transmission of user2/user1, although the transmitted waveforms overlap in both time and frequency. The performance of this system (bit error rate wise) is similar to that of a system in which two bit streams are transmitted through separate channels with same noise power. In [19], it 


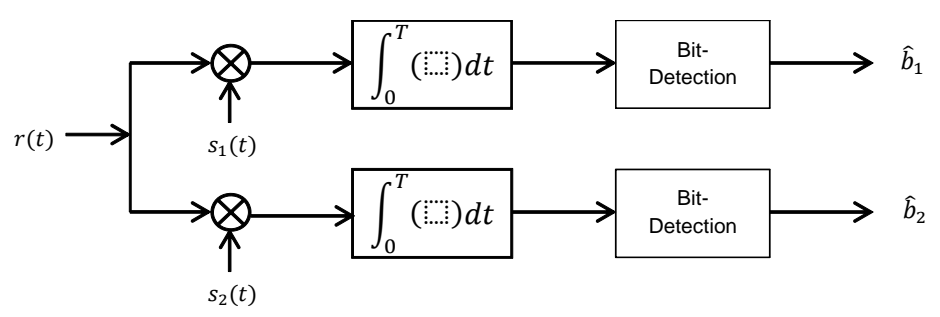

Figure 2.8: Demodulation of transmitted bit streams using correlation detectors. $r(t)$ is the channel output signal.

has been shown that a $K$-user orthogonal CDMA system employing antipodal modulation at the rate of $R$ bits per second requires a bandwidth $B$ approximately equal to

$$
B \approx \frac{R K}{2}
$$

Hence for a given data rate and bandwidth availability, the number of orthogonal signature waveforms will be given by,

$$
K \approx \frac{2 B}{R}
$$

The number of orthogonal signature waveforms is restricted by the available bandwidth and the data rate. However the orthogonality of the signature waveforms is not a mandatory in CDMA. For instance, one can compromise the performance of the system by allowing nonorthogonal interfering users. However the loss of performance can be kept to a tolerable level by carefully selecting the non-orthogonal signature waveforms [20]. The selection of non-orthogonal signatures overcomes the issue of constrained number of simultaneous users. One major benefit of non-orthogonal CDMA is that its reliability depends on the number of simultaneous users, rather than on the potential users. Therefore the performance of the system can be compromised to increase the capacity. This property can benefit WSNs considered in this thesis. Therefore, the number of users viable in a CDMA system is determined by the bandwidth of the channel and the data rate. The other factors that affect a CDMA system are

1. received signal-to-noise ratio, 
2. signature waveform correlation, and

3. redundancy in user data.

In this thesis, dependency of the performance of the proposed decoding scheme on the above factors will be examined.

\subsection{Multi-user detection}

Multi-user detection can be defined as the separation of user data at the receiver. In this thesis, the transmission channel is modeled by a Gaussian MAC (GMAC). The CDMA channel is assumed to be synchronous (i.e. the data from all users arrives at the receiver at the same instant of time). We start by briefly discussing the conventional correlation detector [5].

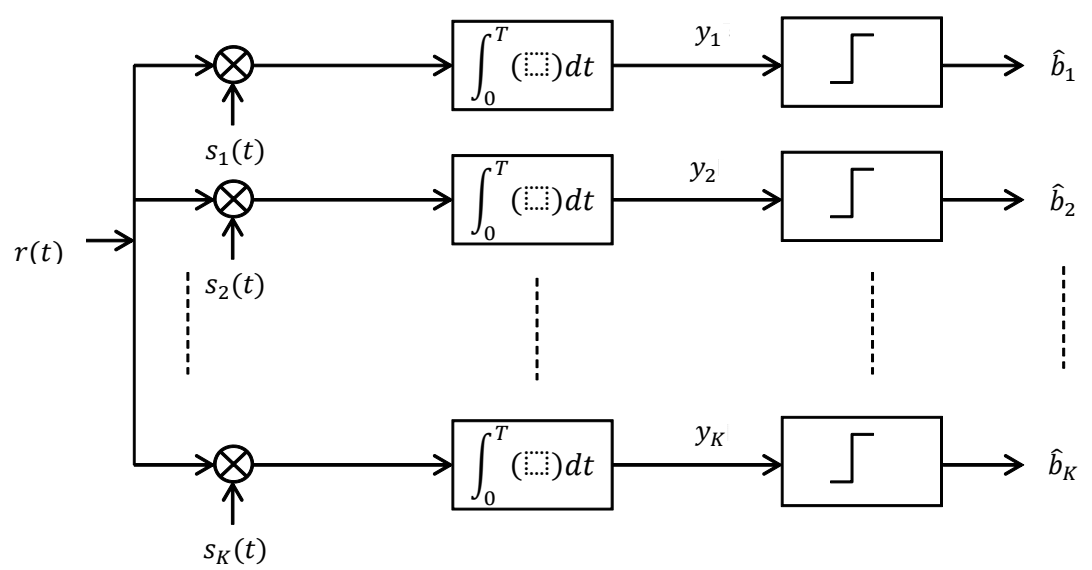

Figure 2.9: Conventional correlation multi-user detector.

The simplest way to separate a user bit stream is to send the received signal $r(t)$ through a set of correlators (or equivalently a set of matched filters) as shown in Figure 2.9. The demodulation of each user (the bit estimation) is carried out independently from the other users. Hence the correlators are known to be single-user correlators. Here, the receiver requires the knowledge of signature waveforms $s_{k}(t)$ and the timing of each user. The major 
issue with this detection method is that it does not take multiple access interference (MAI) into account. To build a detector with improved performance (lower bit error probability), MAI needs to be modeled accurately. The above conventional detector does not use the knowledge other than the signature waveforms and timing of the users. However, with the additional knowledge of the statistical properties of noise, the detector performance can be further improved. The MUD which yields the minimum achievable probability of error was proposed by Sergio Verdú in early 1980s [5].

\subsubsection{The optimal multi-user detector}

Before Verdú's landmark work, it was believed that the matched filter detector will approach optimality with the large number of users (central limit theorem shows that MAI term can be approximated by a Gaussian random variable for large number of users). Even if the MAI term was Gaussian, it does not make the matched filter detector the optimum since the demodulation of each user is carried out independently. More explicitly, for $j^{\text {th }}$ user, $y_{j}$ is not a sufficient statistic for $b_{j}$, but $\left[y_{1}, \ldots, y_{K}\right]^{T}$ is a sufficient statistic for $\left[b_{1}, \ldots, b_{K}\right]^{T}$. In [5], the optimum MUD is obtained under the assumptions that the receiver not only knows the signature waveform and timing of every active user, but it also knows the received amplitudes of all the users and their noise levels.

\section{Individually optimum MUD}

Considering the simple 2-user case

$$
r(t)=A_{1} b_{1} s_{1}(t)+A_{2} b_{2} s_{2}(t)+n(t), \quad 0 \leq t<T_{b} .
$$

The optimum estimate of $b_{1}$ will minimize the probability of error and is obtained by choosing $\hat{b}_{1} \in\{-1,+1\}$ such that a posteriori probability $P\left(b_{1}=\hat{b}_{1} \mid r(t), 0 \leq t<T_{b}\right)$ is maximized. Similarly, the bit estimation for user2 is carried out. 


\section{Jointly optimum MUD}

The individually optimum MUD is not truly optimum since $b_{1}$ and $b_{2}$ are not independent conditioned on the received signal $r(t)$ [5]. Here, the joint a posteriori probability $P\left(b_{1}=\right.$ $\left.\hat{b}_{1}, b_{2}=\hat{b}_{1} \mid r(t), 0 \leq t<T_{b}\right)$ needs to be maximized. However, both types of decisions will agree with a high probability unless the signal-to-noise ratio is very low. This 2user decision making can be extended to $K$-user general case [5]. In [5], it is shown that maximization problem for the $K$-user system can be done on a tree structure that takes $O\left(2^{K}\right)$ operations. Hence the computational complexity grows exponentially with the number of users. Therefore, for a multi-user system with a large number of users a suboptimal system with tractable computational complexity may have to be adopted. However, keeping the loss of performance due to sub-optimality is a key issue. 


\section{Chapter 3}

\section{Iterative Joint Source Channel Decoder}

A $K$ sensor (user) WSN over a synchronous CDMA channel is considered. As mentioned in Chapter 2, the computational complexity of the optimal joint-source-channel (JSC) decoder is exponential in terms of number of users $K$ [21], which is indeed prohibitive for WSNs with medium to large number of users. This turns our heads to consider efficient sub-optimal techniques. In this thesis, a new low-complexity JSC decoder is proposed which uses multi-user detection and VQ decoding in tandem [22]. A similar sub-optimal approach was previously proposed in [23]. However, in [23] VQ decoding is performed using individual decoders for each user, hence the inter-source correlation at the VQ decoding level has not been considered. In contrast to [23], the proposed iterative JSC decoder uses a joint VQ decoder, which takes inter-source correlation in to account. However, such joint VQ decoding can be computationally more complex than separate-individual VQ decoding in [23] (since a joint VQ decoder consider a joint pdf whereas separate-individual VQ decoders consider only a marginal pdf). As will be seen in section 3.3, in the proposed JSC decoder, the computational complexity of the joint VQ decoder is controlled by adopting certain assumptions. The rest of this chapter is dedicated to describing the proposed iterative decoder. 


\subsection{Problem description}

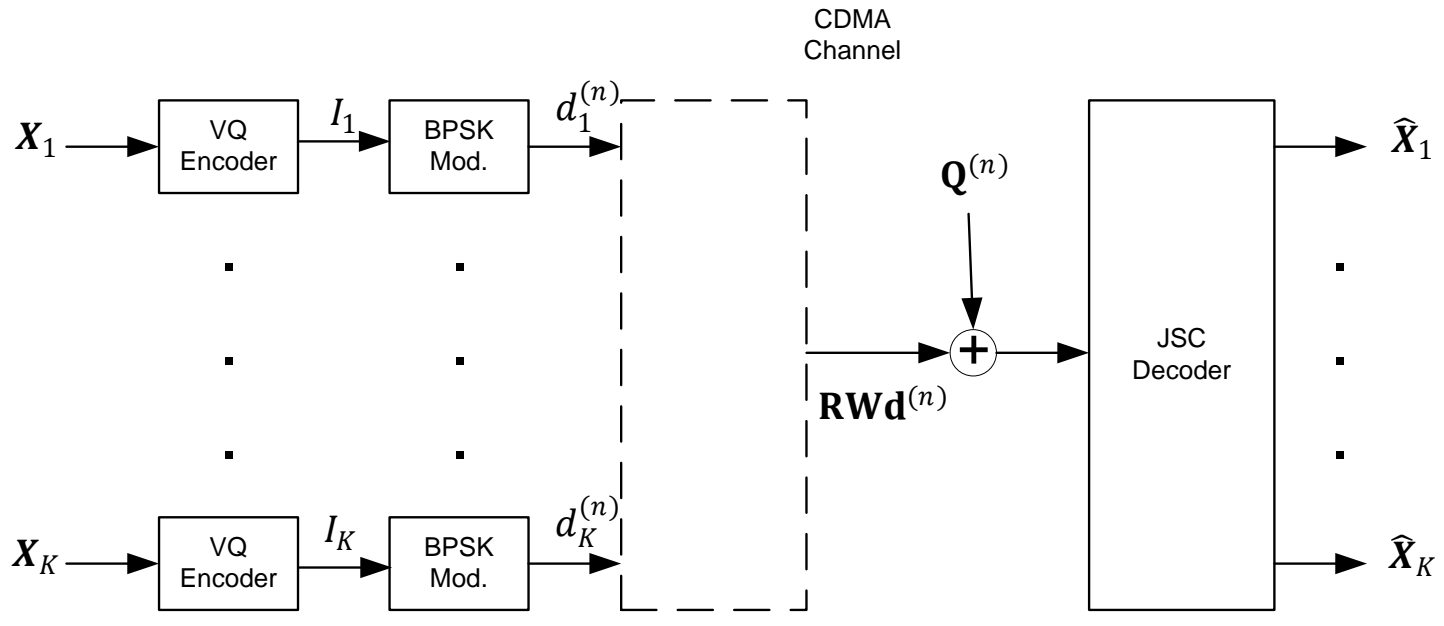

Figure 3.1: The WSN model considered in this thesis.

Consider a WSN with $K$ sensors as shown in Figure 3.1. The sensors are assumed to observe a correlated random field $\left(X_{1}, X_{2}, \ldots, X_{K}\right)$ where $X_{k} \in \mathbb{R}$. Then the sensor $k$ encodes a vector $\mathbf{X}_{k}$ of dimension $d$ (formed by $d$ consecutive samples of the random variable $X_{k}$ ) [17] in to a quantization index $I_{k} \in\{0,1, \ldots, N-1\}$ (where $N=2^{L}$ for some integer $\mathrm{L}$ ). Hence, the rate of the VQ encoder is $r=L / d$ (bits per sample). The $k$-th encoder is described by a partition $\left\{R_{i}^{(k)}\right\}_{i=0}^{N}$ of the Euclidean source space $\mathbb{R}^{d}$ such that $\mathbf{X}_{k} \in R_{i}^{(k)} \Rightarrow I_{k}=i$. For transmission, the index $I_{k}$ is converted into a block represented by the vector $\left.\mathbf{d}_{\mathbf{k}}=\left[d_{k}^{(0)}, \ldots, d_{k}^{(L-1)}\right)\right]^{T}$ of $L$ bits in binary phase-shift keying (BPSK) format (i.e. $b_{k}^{(n)} \in\{ \pm 1\}$ ) using the natural binary representation of the integer $I_{k}$ with logical "zero" corresponding to $d_{k}^{(n)}=1$. The $L$ bits of the user index are tranmitted over a symbol synchronous CDMA channel. Thus, the channel output at the receiver end (outputs from bank of cross-correlators, described in Sec. 2.2.2), $\mathbf{Y}^{(n)}=\left[Y_{1}^{(n)}, \ldots, Y_{K}^{(n)}\right]$, at time $n$ can be expressed as [5, Sec. 2.9.1]

$$
\mathbf{Y}^{(n)}=\mathbf{R W} \mathbf{d}^{(n)}+\mathbf{Q}^{(n)}
$$

where $\mathrm{R}$ is a $K \times K$ matrix consisting of cross correlation between the spreading waveforms 
of the individual sensors, and $\mathbf{W}=\operatorname{diag}\left(W_{1}, W_{2}, \ldots, W_{K}\right)$ is a diagonal matrix of the received sensor amplitude $W_{k}$. The channel noise term $Q^{(n)}$ is zero mean Gaussian with covariance matrix $\sigma^{2} \mathbf{R}$ [5, Sec. 2.9.1]. Let $\hat{\mathbf{X}}_{k} \in \mathbb{R}^{d}$ be the reconstructed vector of $\mathbf{X}_{k}$ using some decoder. Then, the average distortion $D_{\text {sys }}$ of the system is measured using the MSE,

$$
D_{\text {sys }}=\sum_{k=1}^{K} E\left\{\left\|\mathbf{X}_{k}-\hat{\mathbf{X}}_{k}\right\|^{2}\right\} .
$$

Based on the channel outputs $\left\{\mathbf{Y}^{(n) T}\right\}_{n=0}^{L-1}$, the decoder needs to estimate the transmitted source vectors $\left\{\mathbf{X}_{k}\right\}_{k=1}^{K}$. The system considered in this thesis does not use any explicit channel coding. Instead, the receiver performs joint source-channel decoding to mitigate the channel noise and multiuser interference by exploiting inter-source correlation. Depending on the method for processing of $\left\{\mathbf{Y}^{(n) T}\right\}_{n=0}^{L-1}$ different decoders can be obtained.

\subsection{The MMSE decoder}

The MMSE decoder minimizes the distortion $D_{k}=E\left\{\left\|\mathbf{X}_{k}-\hat{\mathbf{X}}_{k}\right\|^{2}\right\}$ for each user $k$. Hence, from estimation theory [24], the optimal estimate $\hat{\mathbf{X}}$ of the source vector $\mathbf{X}$ is the conditional average given by

$$
\hat{\mathbf{X}}_{k}=E\left\{\mathbf{X}_{k} \mid \underline{\mathbf{Y}}=\underline{\mathbf{y}}\right\}, \quad k=1, \ldots K .
$$

It is straight forward to show that, using the Bayes' rule, and the fact that given $\mathbf{I}, \mathbf{X}_{k}$ is independent of $\underline{\mathbf{Y}}$,

$$
E\left\{\mathbf{X}_{k} \mid \underline{\mathbf{Y}}=\underline{\mathbf{y}}\right\}=\sum_{\mathbf{i}} E\left\{\mathbf{X}_{k} \mid \mathbf{I}=\mathbf{i}\right\} P(\mathbf{I}=\mathbf{i} \mid \underline{\mathbf{Y}}=\underline{\mathbf{y}}) .
$$

As a simplification, we make the reasonable assumption that

$$
\begin{aligned}
E\left\{\mathbf{X}_{k} \mid \mathbf{I}=\mathbf{i}\right\} & \approx E\left\{\mathbf{X}_{k} \mid I_{k}=i\right\} \\
& =\mathbf{c}_{i, k}
\end{aligned}
$$


where $c_{i, k}=E\left\{\mathbf{X}_{k} \mid I_{k}=i\right\}$ is the $i$ th centroid of VQ encoder of user $k$, and $\underline{\mathbf{y}}=$ $\left[\mathbf{y}^{(0) T}, \ldots, \mathbf{y}^{(0) T}\right]^{T}$. Then by substituting (3.4) in (3.3) we have

$$
\hat{\mathbf{X}}_{k} \approx \sum_{i=0}^{2^{L}-1} \mathbf{c}_{i, k} P\left(I_{k}=i \mid \underline{\mathbf{Y}}=\underline{\mathbf{y}}\right) .
$$

Note that the estimate in (3.5) is a continuous function of $\underline{\mathbf{y}}$. However, if the CDMA channel is noiseless, the centroids $\mathbf{c}_{i, k}$ are the reconstruction vectors of the MMSE decoder. For

a noiseless channel (or when the channel SNR is very high), the noise vector $\mathbf{Q}^{(n)}=\underline{\mathbf{0}}$, hence the channel input bits can be exactly decided at the receiver-end, regardless of the correlation matrix $\mathbf{R}$. However, the correlation matrix $\mathbf{R}$ becomes relevant to the performance of the decoder in the presence of channel noise [5].

The evaluation of $P\left(I_{k}=i \mid \underline{\mathbf{Y}}=\underline{\mathbf{y}}\right)$ in (3.5) has a computational complexity which grows exponentially with $K$ [21]. If the signature waveforms are orthogonal, the $\mathbf{R}$ matrix becomes the identity matrix. This property itself does not reduce the computational complexity of the decoder. However, if the quantized outputs of the VQ encoders can be modeled as a Markov-random field (MRF), then the $P\left(I_{k}=i \mid \underline{\mathbf{Y}}=\underline{\mathbf{y}}\right)$ can be evaluated with a computational complexity that grows only linearly with the number of sensors $K$ [9]. In the following, an extension to the joint VQ decoder in [9] to accomplish a low-complexity iterative JSC deocder for a non-orthogonal CDMA channel is proposed.

\subsection{The proposed Iterative JSC decoder}

As mentioned earlier, in order to obtain a low-complexity approximation to the optimum MMSE decoder, the approach of performing multi-user detection and VQ decoding in tandem is adopted. The MUD performs the separation of user data based on the CDMA channel outputs, which are effected by multi-user interference due to the non-orthogonal CDMA signatures and the additive channel noise. As it will be demonstrated in Chapter 4, depending on the CDMA signatures used, the multi-user interference among correlated users can be either constructive or destructive. Constructive interference will increase the 


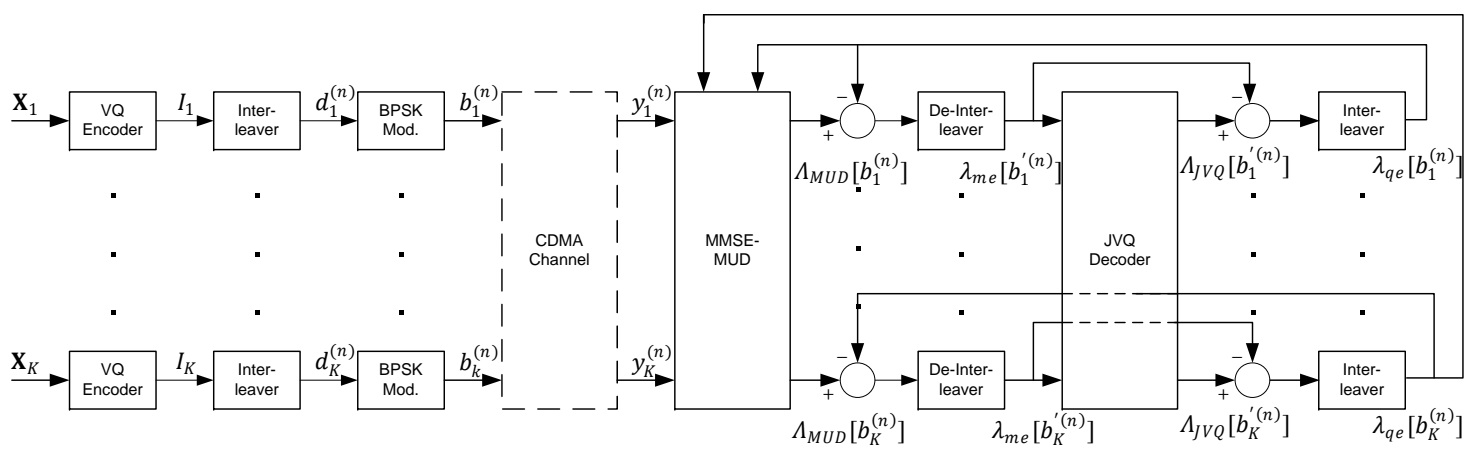

Figure 3.2: Block diagram of the proposed iterative JSC decoder for correlated sources.

available information for the decoding at the channel output, whereas destructive interference will diminish the available information at the channel output. A further discussion on separability of correlated data in MACs appears in $[11,25]$. While the choosing of an optimal set of CDMA signatures for correlated sources is not addressed here, the importance of such optimization will be demonstrated in Chapter 4. One key objective of this design, is to achieve robustness against the effects of the CDMA cross-correlation matrix. This is achieved by using bit-level interleaving for each user, which makes the CDMA channel outputs independent among different users. Because of the interleaving, the MUD can not directly account for inter-source correlation. However, as it will be seen in the next section, the interference cancellation in the MUD is improved by using the extrinsic information computed in the JVQ decoder which exploits the inter-source correlation. The extrinsic information in soft-bit estimates computed by the MUD are extracted and delivered to the JVQ decoder. The MUD and the JVQ decoder interchange extrinsic information about the transmitted bits within iterations and as the improvement through iterations diminishes, the estimates of $\left\{\mathbf{X}_{k}\right\}_{k=1}^{K}$ are computed at the JVQ decoder. The block diagram of the proposed decoder is shown in Figure 3.2. The work related to iterative multi-user detection in channel coded CDMA systems with independent sources can be found in [26-28]. In contrast to communication model used in this thesis, in such coded CDMA systems, the redundancies contained in the channel code words are exploited. Also iterative approaches to JSC decoding in coded independent channels appears in $[29,30]$, in which the redun- 
dancies in the channel code words and in the VQ encoder indexes (due to the dependency among subsequent samples) are exploited alternatively at source and channel decoders. In the following sections, the iterative JSC decoder proposed in this thesis is discussed in detail.

\subsubsection{A soft MUD with interference cancellation}

The optimal MUD discussed in Chapter 2 has a computational complexity that grows exponentially with number of sensors $K$. Therefore, the soft-input soft-output (SISO) MMSE MUD proposed in [26], whose computational complexity is only $O\left(K^{2}\right)$ is adopted. Suppose that interleaver depth is $M L$ bits, for some integer $\mathrm{M}$, which should be large enough to eliminate the inter-bit correlation among the channel input bits from different users. Hence a sequence of $M L$ bits $\left(d_{k}^{(0)}, \ldots, d_{k}^{(M L-1)}\right)$ obtained by quantizing $M$ consecutive input vectors in the sensor $k$ are interleaved to generate a sequence of CDMA channel input bits $\left(b_{k}^{(0)}, \ldots, b_{k}^{(M L-1)}\right)$.

In [26], multi-user detection is done in two stages. In the first stage, the soft interference cancellation is performed on the channel output $\mathbf{y}^{(n)}=\left[y_{1}^{(n)}, \ldots, y_{K}^{(n)}\right]$ for each sensor $k$ at time $n$, based on the extrinsic information delivered by the JVQ decoder. As will be seen in the next section, the extrinsic information is the a prori information about $b_{k}^{(n)}$ obtained from the soft bit estimation of the other bits, $\left\{b_{k}^{(m)}\right\}_{m \neq n}$, based on the factor graph constructed at the JVQ decoder. In [26], the soft interference cancellation uses the soft estimates of channel input bits, which is defined as

$$
\tilde{b}_{k}^{(n)} \triangleq \sum_{b_{k} \in\{+1,-1\}} b_{k} P\left[b_{k}^{(n)}=b_{k}\right] .
$$

The soft bit estimates in a given iteration are computed using the extrinsic prior information computed by the JVQ decoder in the previous iteration. The extrinsic information is the a priori log-likelihood ratio (LLR) given by

$$
\lambda_{q e}^{p}\left[b_{k}^{(n)}\right]=\ln \left(\frac{P\left[b_{k}^{(n)}=+1\right]}{P\left[b_{k}^{(n)}=-1\right.}\right) .
$$


The superscript ${ }^{p}$ indicates the quantity computed in the previous iteration. After some mathematical manipulations, the prior probability $P\left[b_{k}^{(n)}=b_{k}\right]$, for $b_{k} \in\{+1,-1\}$, can be written as

$$
\begin{aligned}
P\left[b_{k}^{(n)}=b_{k}\right] & =\frac{\exp \left(b_{k} \lambda_{q e}^{p}\left[b_{k}^{(n)}\right]\right)}{1+\exp \left(b_{k} \lambda_{q e}^{p}\left[b_{k}^{(n)}\right]\right)} \\
& =\frac{\exp \left(\frac{1}{2} b_{k} \lambda_{q e}^{p}\left[b_{k}^{(n)}\right]\right)}{\exp \left(-\frac{1}{2} b_{k} \lambda_{q e}^{p}\left[b_{k}^{(n)}\right]\right)+\exp \left(\frac{1}{2} b_{k} \lambda_{q e}^{p}\left[b_{k}^{(n)}\right]\right)}
\end{aligned}
$$

Using Euler's formula and hyperbolic function definitions, (3.8) can be written as

$$
\begin{aligned}
P\left[b_{k}^{(n)}=b_{k}\right] & =\frac{\cosh \left(\frac{1}{2} b_{k} \lambda_{q e}^{p}\left[b_{k}^{(n)}\right]\right)\left[1+b_{k} \tanh \left(\frac{1}{2} b_{k} \lambda_{q e}^{p}\left[b_{k}^{(n)}\right]\right)\right]}{2 \cosh \left(\frac{1}{2} b_{k} \lambda_{q e}^{p}\left[b_{k}^{(n)}\right]\right)} \\
& =\frac{1}{2}\left[1+b_{k} \tanh \left(\frac{1}{2} b_{k} \lambda_{q e}^{p}\left[b_{k}^{(n)}\right]\right)\right] .
\end{aligned}
$$

By substituting (3.9) into (3.6), we have

$$
\tilde{b}_{k}^{(n)}=\tanh \left(\frac{1}{2} \lambda_{q e}^{p}\left[b_{k}^{(n)}\right]\right), \quad k=1, \ldots, K .
$$

Note that for the first iteration, soft interference canceler assumes all the channel input bits to be equally likely, and hence the $\lambda_{q e}^{p}\left[b_{k}^{(n)}\right]=0$, for $1 \leq k \leq K$ and $0 \leq n \leq M L-1$. Then these soft bit estimates are used to cancel the interference in the user $k$, by performing interference cancellation on the CDMA channel output $\mathbf{y}^{(n)}$ at time $n$. The interference canceled vector $\tilde{\mathbf{y}}_{k}^{(n)}$ for the sensor $k$ is obtained

$$
\tilde{\mathbf{y}}_{k}^{(n)}=\mathbf{y}^{(n)}-\mathbf{R W} \tilde{\mathbf{b}}_{k}^{(n)}
$$

where $\tilde{\mathbf{b}}_{k}^{(n)}=\left[\tilde{b}_{1}^{(n)}, \ldots, \tilde{b}_{k-1}^{(n)}, 0, \tilde{b}_{k+1}^{(n)}, \ldots, \tilde{b}_{K}^{(n)}\right]$. In [26], a linear MMSE filter is applied to $\tilde{\mathbf{y}}_{k}^{(n)}$, in order to further suppress the residual interference in $\tilde{\mathbf{y}}_{k}^{(n)}$. The linear MMSE filter $a_{k}^{(n)}$ in [26] minimizes the mean square error between the CDMA channel input $b_{k}^{(n)}$ and the filter output $u_{k}^{(n)}$

$$
u_{k}^{(n)}=a_{k}^{(n) T} \tilde{\mathbf{y}}_{k}^{(n)}
$$


where $\mathbf{a}_{k}^{(n)} \in \mathbb{R}^{K}$ is the filter coefficient vector, derived using the correlation matrix $\mathbf{R}$, the diagonal matrix $\mathbf{W}$ with received sensor amplitudes, and the soft estimates of the other bits at time $n,\left\{\tilde{b}_{j}^{(n)}\right\}_{j \neq k}$. From [26], it follows that for the sensor $k$ at time $n$,

$$
\mathbf{a}_{k}^{(n)}=\left[\mathbf{R V}_{k}^{(n)} \mathbf{R}\right]^{-1} \mathbf{R W} \mathbf{e}_{k}
$$

where $V_{k}^{(n)}$ is defined as

$$
V_{k}^{(n)} \triangleq \mathbf{W} \operatorname{cov}\left\{\mathbf{b}^{(n)}-\tilde{\mathbf{b}}_{k}^{(n)}\right\} \mathbf{W}
$$

and, $\operatorname{cov}\left\{\mathbf{b}^{(n)}-\tilde{\mathbf{b}}_{k}^{(n)}\right\}$ is the covariance matrix of vector $\mathbf{b}^{(n)}=\left[b_{1}^{(n)}, \ldots, b_{K}^{(n)}\right]$. It can be shown that $V_{k}^{(n)}$ is given by [26]

$$
V_{k}^{(n)}=\sum_{j \neq k} W_{j}^{2}\left[1-\tilde{b}_{j}^{(n) 2}\right] \mathbf{e}_{j} \mathbf{e}_{j}^{T}+W_{k}^{2} \mathbf{e}_{k} \mathbf{e}_{k}^{T}
$$

where $\mathbf{e}_{k}$ denotes a $K$-vector of all zeros, except for the $k$-th element, which is 1 . It is clear that from (3.11) and (3.13), the a priori information is vital for both interference cancellation and MMSE filtering.

\section{Gaussian approximation of soft MMSE filter output}

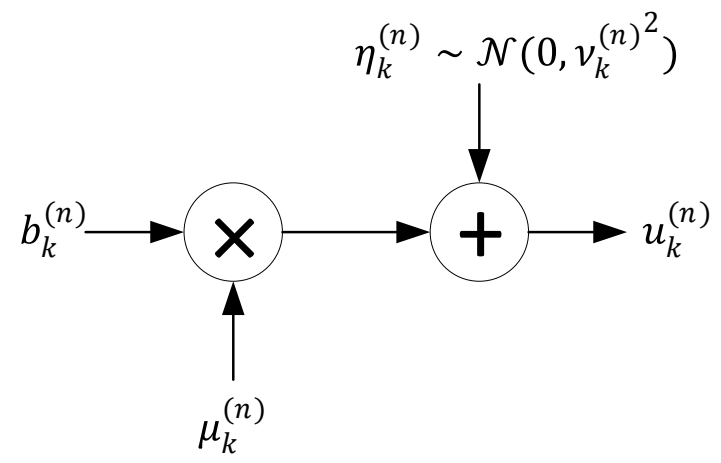

Figure 3.3: The equivalent AWGN channel.

It is shown in [31] that the distribution of the residual interference-plus-noise at the output of a linear MMSE-MUD is well approximated by a Gaussian distribution. As shown in Figure 3.3, the MMSE MUD output $u_{k}^{(n)}$ can be assumed to represent the output of an 
equivalent AWGN channel having $b_{k}^{(n)}$ as the input symbol. This equivalent channel is modeled by

$$
u_{k}^{(n)}=\mu_{k}^{(n)} b_{k}^{(n)}+\eta_{k}^{(n)}
$$

where the parameter $\mu_{k}^{(n)}$ is the equivalent amplitude of the $k$ th sensor's signal, and $\eta_{k}^{(n)} \sim$ $\mathcal{N}\left(0,\left(\nu_{k}^{(n)}\right)^{2}\right)$ is a Gaussian noise sample. The expression for parameters $\mu_{k}^{(n)}$ and $\left(\nu_{k}^{(n)}\right)^{2}$ are shown to be [26]

$$
\mu_{k}^{(n)}=W_{k}^{2}\left[\left[\mathbf{V}_{k}^{(n)}+\sigma^{2} \mathbf{R}\right]^{-1}\right]_{k k}
$$

and

$$
\left(\nu_{k}^{(n)}\right)^{2}=\mu_{k}^{(n)}-\left(\mu_{k}^{(n)}\right)^{2} .
$$

The a posteriori LLR of $b_{k}^{(n)}$ at the output of the MMSE MUD is given by

$$
\Lambda_{M U D}\left[b_{k}^{(n)}\right] \triangleq \ln \left(\frac{P\left[b_{k}^{(n)}=+1 \mid u_{k}^{(n)}\right]}{P\left[b_{k}^{(n)}=-1 \mid u_{k}^{(n)}\right]}\right) .
$$

Using the Bayes' rule, (3.19) can be written as

$$
\Lambda_{M U D}\left[b_{k}^{(n)}\right]=\underbrace{\ln \left(\frac{P\left[u_{k}^{(n)} \mid b_{k}^{(n)}=+1\right]}{P\left[u_{k}^{(n)} \mid b_{k}^{(n)}=-1\right]}\right)}_{\lambda_{m e}\left[b_{k}^{(n)}\right]}+\underbrace{\ln \left(\frac{P\left[b_{k}^{(n)}=+1\right]}{P\left[b_{k}^{(n)}=-1\right]}\right)}_{\lambda_{q e}^{p}\left[b_{k}^{(n)}\right]},
$$

where the second term in (3.20), represents the a priori LLR of the channel input bit $b_{k}^{(n)}$, which is computed by the JVQ decoder in the previous iteration, interleaved and then fed back to the MMSE-MUD. The first term in (3.20) denoted by $\lambda_{m e}\left[b_{k}^{(n)}\right]$ represents the extrinsic information delivered by the MMSE-MUD. From (3.16) the extrinsic information 
delivered by the MMSE-MUD is simplified as

$$
\begin{aligned}
\lambda_{m e}\left[b_{k}^{(n)}\right] & \triangleq \ln \left(\frac{P\left[u_{k}^{(n)} \mid b_{k}^{(n)}=+1\right]}{P\left[u_{k}^{(n)} \mid b_{k}^{(n)}=-1\right]}\right) \\
& =-\frac{\left[u_{k}^{(n)}-\mu_{k}^{(n)}\right]^{2}}{2\left(\nu_{k}^{(n)}\right)^{2}}+\frac{\left[u_{k}^{(n)}-\mu_{k}^{(n)}\right]^{2}}{2\left(\nu_{k}^{(n)}\right)^{2}} \\
& =\frac{2 \mu_{k}^{(n)} u_{k}^{(n)}}{\left(\nu_{k}^{(n)}\right)^{2}} \\
& =\frac{2 u_{k}^{(n)}}{1-\mu_{k}^{(n)}}
\end{aligned}
$$

The extrinsic information $\lambda_{m e}\left[b_{k}^{(n)}\right]$ which is not influenced by the a priori information $\lambda_{q e}^{p}\left[b_{k}^{(n)}\right]$ delivered by JVQ decoder, is then de-interleaved and fed in to JVQ decoder, as the a priori information in the next iteration.

\subsubsection{Optimal JVQ decoder}

The sequence of linear MMSE filter outputs $u_{k}^{(0)}, \ldots, u_{k}^{(L-1)}$ from the AWGN channel in (3.16), for each sensor $k=1, \ldots, K$ is de-interleaved to form the input to the JVQ decoder. Therefore, for the VQ index $I_{k}$ transmitted by the sensor $k$, the JVQ decoder receives the vector $\mathbf{Y}^{\prime}{ }_{k}=\left[Y_{k}^{\prime(0)}, \ldots, Y_{k}^{\prime(L-1)}\right]^{T} \in \mathbb{R}^{L}$. The MMSE optimal outputs $\left\{\hat{\mathbf{X}}_{k}\right\}_{k=1}^{K}$, can thus be computed by (3.5), but with $\underline{\mathbf{Y}}$ replaced by the vector $\underline{\mathbf{Y}}^{\prime}=\left[\mathbf{Y}^{\prime}{ }_{1}^{T}, \ldots, \mathbf{Y}^{\prime}{ }_{1}^{T}\right]^{T}$. Now consider

$$
P\left(I_{k}=i_{k} \mid \underline{\mathbf{Y}}^{\prime}=\underline{\mathbf{y}}^{\prime}\right)=\frac{\sum_{\mathbf{I}: I_{k}=i_{k}} p\left(\mathbf{I}, \mathbf{y}^{\prime}\right)}{\sum_{\mathbf{I}} p\left(\mathbf{I}, \mathbf{y}^{\prime}\right)},
$$

where $\mathbf{I}=\left[I_{1}, \ldots, I_{K}\right]$, and using the fact that $\left\{\mathbf{Y}^{\prime}{ }_{k}\right\}_{k=1}^{K}$ are conditionally independent given $\mathbf{I}, p\left(\mathbf{I}=\mathbf{i}, \underline{\mathbf{Y}}^{\prime}=\underline{\mathbf{y}}^{\prime}\right)$ can be written as

$$
p\left(\mathbf{I}=\mathbf{i}, \underline{\mathbf{Y}}^{\prime}=\underline{\mathbf{y}}^{\prime}\right)=\prod_{k=1}^{K} p\left(\mathbf{y}_{k}^{\prime} \mid \mathbf{I}=\mathbf{i}\right) P(\mathbf{I}=\mathbf{i}) .
$$

The pmf $P(\mathbf{I})$ in (3.23) can be written as

$$
p(\mathbf{I})=\prod_{k=1}^{K} P\left(I_{k} \mid I_{1}, \ldots, I_{k-1}\right) .
$$


Notice that the $I_{k}$ is the VQ encoder output of k-th sensor observation. In most practical situations, the correlation between two sensor observations decays exponentially with the distance, which restricts the conditional dependency in (3.24) into a smaller subset (see [9]). For simplicity, assume that the sensors are in a linear array. Then it is reasonable to assume that

$$
P\left(I_{k} \mid I_{1}, \ldots, I_{k-1}\right) \approx P\left(I_{k} \mid I_{k-1}\right)
$$

In this case, $\mathbf{I}$ is a first-order MRF. Now the joint $\operatorname{pmf} p(\mathbf{I})$ can be written as

$$
p(\mathbf{I})=\prod_{k=1}^{K} P\left(I_{k} \mid I_{k-1}\right) \text {. }
$$

Substituting (3.26) into (3.23), we have

$$
p\left(\mathbf{I}=\mathbf{i},{\underline{\mathbf{Y}^{\prime}}}^{\prime}=\underline{\mathbf{y}}^{\prime}\right)=\prod_{k=1}^{K} p\left(\mathbf{y}_{k}^{\prime} \mid \mathbf{I}=\mathbf{i}\right) P\left(I_{k}=i_{k} \mid I_{k-1}=i_{k-1}\right) .
$$

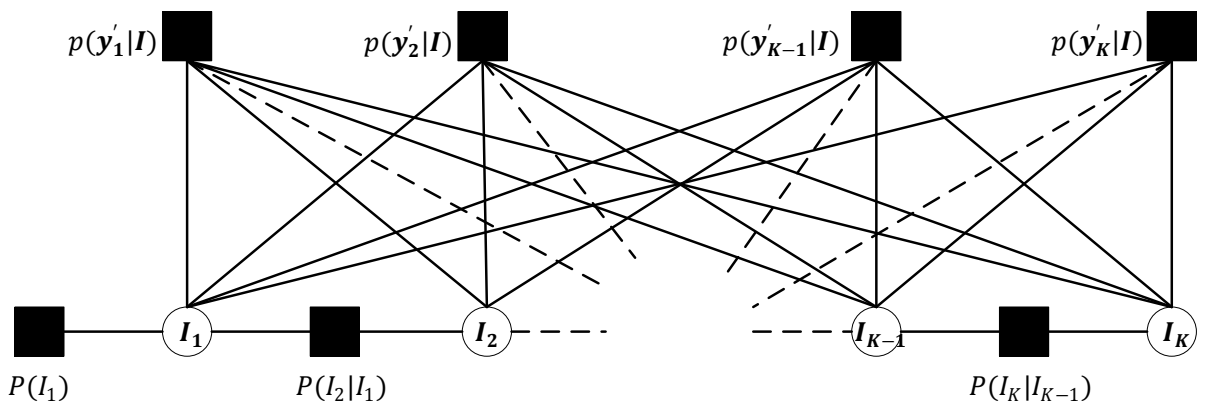

Figure 3.4: The factor-graph representation of joint pdf in (3.27) .

The evaluation of (3.22) may be done more efficiently by factorizing the $p\left(\mathbf{I}=\mathbf{i}, \underline{\mathbf{Y}}^{\prime}=\right.$ $\left.\underline{\mathbf{y}}^{\prime}\right)$, and applying a suitable algorithm which exploits the way the "global" function is factorized into a product of less complex "local" functions. Such a factorization can be visualized using a factor graph, a bipartite graph that expresses which variables are arguments of which local functions. The sum-product algorithm (SPA) $[8,9,32]$ is a widely used algorithm to evaluate marginal functions which operates in a factor graph to evaluate marginal functions associated with the global function. The factor-graph representation of joint pdf 
$p\left(\mathbf{I}=\mathbf{i}, \underline{\mathbf{Y}}^{\prime}=\underline{\mathbf{y}}^{\prime}\right)$ is shown in Figure 3.4, which can be used to evaluate the marginal probabilities given by (3.22) using the SPA. The computational complexity of the SPA is determined by the number of edges incident upon a variable node in the factor-graph. The computational complexity for the factor-graph shown in Figure 3.4 grows exponentially with the number of variable nodes $K\left(I_{1}, \ldots, I_{K}\right)$, as every factor node $p\left(\mathbf{y}_{k}^{\prime} \mid \mathbf{I}\right)$ connects to all variable nodes. In order to make that the complexity of the JVQ decoder linear with the number of users, assume that MMSE-MUD presents orthogonal channels to the VQ decoder, as considered in $[8,9,33]$. This is also a reasonable assumption, as iterative message passing between the MMSE-MUD and joint VQ decoder will minimize the performance degradation due to mutual interference. With this assumption (3.27) can be expressed as

$$
p\left(\mathbf{I}=\mathbf{i}, \underline{\mathbf{Y}}^{\prime}=\underline{\mathbf{y}}^{\prime}\right)=\prod_{k=1}^{K} p\left(\mathbf{y}_{k}^{\prime} \mid I_{k}=i_{k}\right) P\left(I_{k}=i_{k} \mid I_{k-1}=i_{k-1}\right) .
$$

In (3.28), the conditional pdf $p\left(\mathbf{y}^{\prime}{ }_{k} \mid \mathbf{I}\right)$ of the AWGN channel output can be written as (using the memoryless property in AWGN channels)

$$
p\left(\mathbf{y}_{k}^{\prime} \mid I_{k}=i_{k}\right)=\prod_{n=0}^{L-1} p\left(y_{k}^{\prime(n)} \mid b_{n}^{\prime}\left(i_{k}\right)\right)
$$

where $b_{n}^{\prime}\left(i_{k}\right) \in\{-1,+1\}$ is the BPSK modulated value of the $n$th binary bit of integer $i_{k}$. Now using the AWGN channel parameters,

$$
p\left(\mathbf{y}_{k}^{\prime} \mid I_{k}=i_{k}\right)=\prod_{n=0}^{L-1} g_{k}^{(n)} \exp \left(\frac{\mu_{k}^{(n)} y_{k}^{\prime(n)} b_{n}^{\prime}\left(i_{k}\right)}{\left(\nu_{k}^{(n)}\right)^{2}}\right)
$$

where $g_{k}^{(n)}=\left(1 / \sqrt{2 \pi} \nu_{k}^{(n)}\right) \exp \left[-\left({y^{\prime}}_{k}^{(n)^{2}}+\mu_{k}^{(n)^{2}}\right) /\left(2\left(\nu_{k}^{(n)}\right)^{2}\right)\right]$. The LLR of the equivalent AWGN channel output $y_{k}^{\prime(n)}$ is given by

$$
\begin{aligned}
\lambda_{m e}^{(p)}\left[b_{n}^{\prime}\left(i_{k}\right)\right] & =\ln \left(\frac{p\left[y_{k}^{\prime(n)} \mid b_{n}^{\prime}\left(i_{k}\right)=+1\right.}{p\left[y_{k}^{\prime(n)} \mid b_{n}^{\prime}\left(i_{k}\right)=-1\right.}\right) \\
& =\frac{2{y_{k}^{\prime}}_{k}^{(n)}}{\left(\nu_{k}^{(n)}\right)^{2}} .
\end{aligned}
$$


By substituting (3.31) in (3.30) we get

$$
p\left(\mathbf{y}_{k}^{\prime} \mid I_{k}=i_{k}\right)=\prod_{n=0}^{L-1} g_{k}^{(n)} \exp \left(\frac{1}{2} b_{n}^{\prime}\left(i_{k}\right) \lambda_{m e}^{(p)}\left[b_{n}^{\prime}\left(i_{k}\right)\right]\right) .
$$

Now using the fact that $\exp (u \theta)=\cosh \theta(1+u \tanh (\theta))$ for $u \in\{-1,+1\}$, (3.32) can be written as

$$
p\left(\mathbf{y}_{k}^{\prime} \mid I_{k}=i_{k}\right)=\prod_{n=0}^{L-1} g_{k}^{(n)} \cosh \left(\frac{1}{2} \lambda_{m e}^{(p)}\left[b_{n}^{\prime}\left(i_{k}\right)\right]\right)\left[1+b_{n}^{\prime}\left(i_{k}\right) \tanh \left(\frac{1}{2} \lambda_{m e}^{(p)}\left[b_{n}^{\prime}\left(i_{k}\right)\right]\right)\right] .
$$

Denote

$$
\phi_{n}\left(i_{k}\right)=\left[1+b_{n}^{\prime}\left(i_{k}\right) \tanh \left(\frac{1}{2} \lambda_{m e}^{(p)}\left[b_{n}^{\prime}\left(i_{k}\right)\right]\right)\right] .
$$

Using the fact that $g_{k}^{(n)} \cosh \left(\frac{1}{2} \lambda_{m e}^{(p)}\left[b_{n}^{\prime}\left(i_{k}\right)\right]\right)$ is a constant with respect to $\mathbf{I}$ and by substituting (3.32) and (3.27) into (3.22) we get

$$
P\left(I_{k}=i_{k} \mid \underline{\mathbf{Y}}^{\prime}=\underline{\mathbf{y}}^{\prime}\right)=\frac{\sum_{\mathbf{I}: I_{k}=i_{k}} \prod_{k=1}^{K}\left(\prod_{n=0}^{L-1} \phi_{n}\left(i_{k}\right)\right) P\left(I_{k}=i_{k} \mid I_{k-1}=i_{k-1}\right)}{\sum_{\mathbf{I}} \prod_{k=1}^{K}\left(\prod_{n=0}^{L-1} \phi_{n}\left(i_{k}\right)\right) P\left(I_{k}=i_{k} \mid I_{k-1}=i_{k-1}\right)} .
$$

Now, the function $\prod_{k=1}^{K}\left(\prod_{n=0}^{L-1} \phi_{n}\left(i_{k}\right)\right) P\left(I_{k}=i_{k} \mid I_{k-1}=i_{k-1}\right)$ is represented in the form of a factor graph (see Figure 3.5). Then the posterior probabilities $P\left(I_{k}=i_{k} \mid \underline{\mathbf{Y}}^{\prime}=\underline{\mathbf{y}}^{\prime}\right)$ for $k=1, \ldots, K$ can be computed using the SPA. Notice that each factor node $\prod_{n=0}^{L-1} \phi_{n}\left(i_{k}\right)$ only connects to a single variable node $\left(I_{k}\right)$ and hence an additional user will add a constant complexity to the computation of any marginal function $P\left(I_{k}=i_{k} \mid \underline{\mathbf{Y}}^{\prime}=\underline{\mathbf{y}}^{\prime}\right)$ (see [32]). Therefore the computational complexity of JVQ decoder grows linearly with number of users $K($ i.e. $O(K)$ ). Note that the overall complexity of the JSC decoder is the addition of computational complexities of the MMSE-MUD and the JVQ decoder. Therefore the total computational complexity of the iterative decoder is $O\left(K^{2}+K\right)$, and for large number of users (i.e. $K>>1$ ) it can be approximated to $O\left(K^{2}\right)$. Also the numerical results suggest that number of iterations required for convergence is a constant, regardless of the number of users $K$, and therefore the iterative process scales the computational complexity by a constant that depends on $K$. The a posteriori LLR of the bit $b_{n}^{\prime}\left(i_{k}\right)$ at the JVQ decoder 

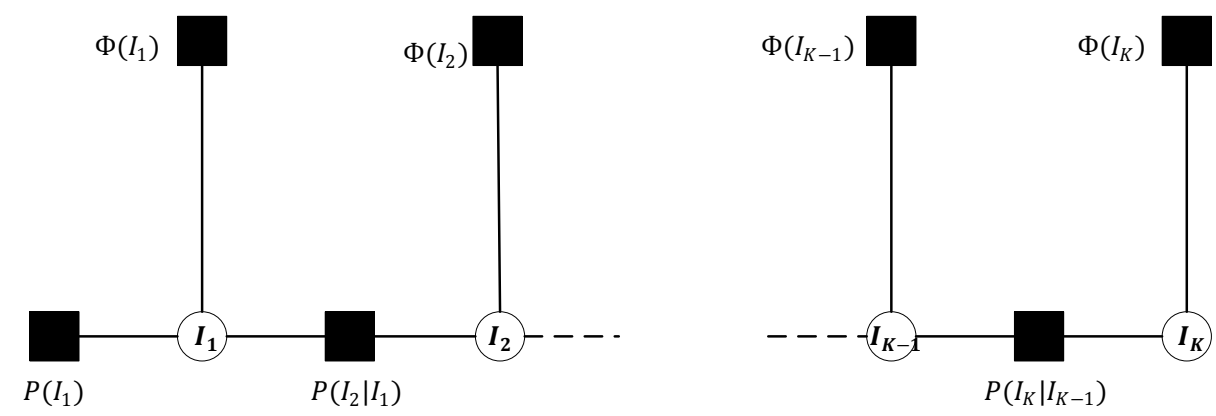

$$
\Phi\left(I_{k}\right)=\prod_{n=0}^{L-1} \phi_{n}\left(I_{k}\right)
$$

Figure 3.5: The factor-graph representation of $\prod_{k=1}^{K}\left(\prod_{n=0}^{L-1} \phi_{n}\left(i_{k}\right)\right) P\left(I_{k}=i_{k} \mid I_{k-1}=i_{k-1}\right)$.

output is given by

$$
\Lambda_{J V Q}\left[b_{n}^{\prime}\left(i_{k}\right)\right]=\ln \left(\frac{p\left[b_{n}^{\prime}\left(i_{k}\right)=+1 \mid \underline{\mathbf{y}}^{\prime}\right]}{p\left[b_{n}^{\prime}\left(i_{k}\right)=-1 \mid \underline{\mathbf{y}}^{\prime}\right]}\right)
$$

where the bit a posteriori probabilities $p\left(b_{n}^{\prime}\left(i_{k}\right) \mid \underline{\mathbf{y}}^{\prime}\right)$ can be computed by marginalizing the index probabilities in (3.22). Using the Bayes' rule and the assumption of orthogonal AWGN channels at the JVQ decoder input, (3.36) can be written as

$$
\Lambda_{J V Q}\left[b_{n}^{\prime}\left(i_{k}\right)\right]=\underbrace{\ln \left(\frac{p\left[y_{k}^{\prime(n)} \mid b_{n}^{\prime}\left(i_{k}\right)=+1\right.}{p\left[y_{k}^{\prime(n)} \mid b_{n}^{\prime}\left(i_{k}\right)=-1\right.}\right)}_{\lambda_{m e}^{p}\left[b_{n}^{\prime}\left(i_{k}\right)\right]}+\underbrace{\ln \left(\frac{P\left[b_{n}^{\prime}\left(i_{k}\right)=+1 \mid \overline{\mathbf{y}}_{(k, n)}^{\prime}\right]}{P\left[b_{n}^{\prime}\left(i_{k}\right)=-1 \mid \overline{\mathbf{y}}_{(k, n)}^{\prime}\right]}\right)}_{\lambda_{q e}\left[b_{n}^{\prime}\left(i_{k}\right)\right]}
$$

where

$$
\begin{aligned}
\overline{\mathbf{y}}_{(k, n)}^{\prime} & =\left[\mathbf{y}_{1}^{\prime T}, \ldots, \mathbf{y}_{k-1}^{\prime T}, \tilde{\mathbf{y}}_{(k)}^{T}, \mathbf{y}_{k+1}^{\prime T}, \ldots, \mathbf{y}_{K}^{\prime T}\right]^{T} \text { and } \\
\tilde{\mathbf{y}}_{(k)}^{\prime} & =\left[y_{k}^{\prime(0)}, \ldots, y_{k}^{\prime(n-1)}, y_{k}^{\prime(n+1)}, \ldots, y_{k}^{\prime(L-1)}\right]^{T} .
\end{aligned}
$$

Thus, the extrinsic LLR of the bit $b_{n}^{\prime}\left(i_{k}\right)$ (which is obtained from other AWGN outputs $\left\{y_{k}^{\prime(m)}\right\}_{m \neq n}$ corresponding to index vector $\mathbf{I}$, by exploiting the constructed factor graph in Figure 3.5) can be computed from JVQ decoder output as

$$
\lambda_{q e}\left[b_{n}^{\prime}\left(i_{k}\right)\right]=\Lambda_{J V Q}\left[b_{n}^{\prime}\left(i_{k}\right)\right]-\lambda_{m e}^{p}\left[b_{n}^{\prime}\left(i_{k}\right)\right]
$$

which is interleaved and fed back to the MUD for interference cancellation in bits, other 
than $b_{n}^{\prime}\left(i_{k}\right)$, see Figure 3.2. Initially there is very little correlation between extrinsic information $\lambda_{m e}^{p}\left[b_{n}^{\prime}\left(i_{k}\right)\right]$ and $\lambda_{q e}\left[b_{n}^{\prime}\left(i_{k}\right)\right]$. Since both MMSE-MUD and JVQ decoder use the same information $\lambda_{m e}^{p}\left[b_{n}^{\prime}\left(i_{k}\right)\right]$ and $\lambda_{q e}\left[b_{n}^{\prime}\left(i_{k}\right)\right]$ become more and more correlated. The iterations will continue until there is no reduction in MSE. 


\section{Chapter 4}

\section{Simulation Results and Discussion}

In this chapter, the simulation results are presented in order to illustrate the performance of the proposed iterative JSC decoder. All the results presented here have been obtained with Lloyd-Max scalar quantization [17] of mean-zero, unit-variance correlated Gaussian sources. First, a four-sensor system as shown in Figure 4.1 is considered, for which case it is feasible to compute the performance of the MMSE decoder given by (3.5). Note that in this case the joint source (VQ) MMSE decoder (JSD) can be implemented without the MRF source-model assumption discussed in Chapter 3. The linear array of sensors are assumed to observe a first-order MRF, $X_{k}$ for $k=1, \ldots, 4$, with the correlation between two nearest observations is 0.9 . Then the source covariance matrix in the four-sensor system under consideration is given by $[\mathbf{C}]_{k, l}=0.9^{|k-l|}$ for $k, l=1, \ldots, 4$. In all simulations, a test data (source samples) set of $10^{5}$ has been used.

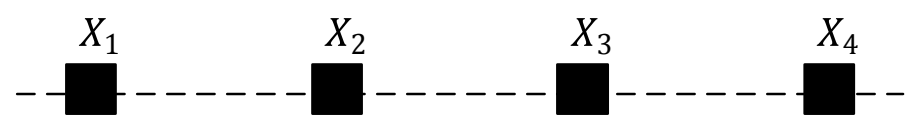

Figure 4.1: The linear array of sensors under consideration. 


\subsection{Performance comparison between iterative and non- iterative JSC decoding}

First, a CDMA channel with the following cross-correlation matrix is considered.

$$
\mathbf{R}_{1}=\left(\begin{array}{cccc}
1 & -0.7 & 0.7 & -0.7 \\
-0.7 & 1 & -0.7 & 0.7 \\
0.7 & -0.7 & 1 & -0.7 \\
-0.7 & 0.7 & -0.7 & 1
\end{array}\right)
$$

Optimum scalar quantizers with the resolution 2 bits/sample are used in all simulations. Notice that the quantizer SNR for a Gaussian source with unit variance is $9.2996 \mathrm{~dB}$. The Figure 4.2 presents the average reconstruction signal-to-noise ratio (RSNR) at the output of different decoders, which is defined by

$$
\mathrm{RSNR} \triangleq \frac{1}{K} \sum_{k=1}^{K} \frac{E\left\{\left\|\mathbf{X}_{k}\right\|^{2}\right\}}{E\left\{\left\|\mathbf{X}_{k}-\hat{\mathbf{X}}_{k}\right\|^{2}\right\}}
$$

The RSNR for each decoder is evaluated at different values of the channel signal-tonoise ratio (CSNR) $W^{2} / \sigma^{2}$, assuming that amplitudes of the all sensor signals are equal (i.e., $W_{k}=W=1$ ). All iterative JSC decoders in Figure 4.2 use interleaving (interleaving depth is 1000 bits) and the decoders without interleaving are explicitly indicated. The plots labeled "initial" refers to the JSC decoders which do not use any iterations. The optimal MUD refers to the maximum-likelihood MUD (see [26, (23)]) which gives an upper-bound to the performance of the linear MMSE-MUD described in Sec. 3.3.1. MMSE-JSD refers to the JVQ decoder described in Sec. 3.3.2. Also note that the MMSE decoder is implemented in this case without the MRF source-model assumption. Note that even though the MMSE decoder is computationally tractable with four users, with large number of users the computational complexity is prohibitive. As can be seen in Figure 4.2, in the low to medium CSNR region the performance of the all non-iterative JSC decoders which do not 
Table 4.1: The RSNR improvement of MMSE MUD + JVQ decoder through iterations. The CDMA crosscorrelation matrix is $\mathbf{R}_{1}$.

\begin{tabular}{ccccccc}
\hline & \multicolumn{7}{c}{ RSNR (dB) with number of iterations } \\
\cline { 2 - 7 } CSNR (dB) & initial & 1 & 2 & 3 & 4 & 5 \\
\hline 0 & 3.5235 & 3.7645 & 3.7801 & 3.7816 & 3.7818 & 3.7818 \\
2 & 4.5682 & 4.9483 & 4.9864 & 4.9900 & 4.9905 & 4.9906 \\
4 & 5.8236 & 6.3028 & 6.3573 & 6.3639 & 6.3647 & 6.3650 \\
6 & 7.1444 & 7.6259 & 7.6656 & 7.6688 & 7.6680 & 7.6676 \\
8 & 8.2312 & 8.5556 & 8.5720 & 8.5709 & 8.5695 & 8.5696 \\
10 & 8.9013 & 9.0358 & 9.0261 & 9.0238 & 9.0234 & 9.0233 \\
\hline
\end{tabular}

Table 4.2: The RSNR improvement of optimum MUD + JVQ decoder through iterations. The CDMA crosscorrelation matrix is $\mathbf{R}_{1}$.

\begin{tabular}{ccccccc}
\hline & \multicolumn{7}{c}{ RSNR (dB) with number of iterations } \\
\cline { 2 - 7 } CSNR (dB) & initial & 1 & 2 & 3 & 4 & 5 \\
\hline 0 & 3.5384 & 3.8244 & 3.8497 & 3.8505 & 3.8506 & 3.8505 \\
2 & 4.6645 & 5.1239 & 5.1710 & 5.1775 & 5.1780 & 5.1782 \\
4 & 6.0325 & 6.6131 & 6.6639 & 6.6709 & 6.6723 & 6.6721 \\
6 & 7.4792 & 8.0500 & 8.1060 & 8.1094 & 8.1111 & 8.1112 \\
8 & 8.5511 & 8.8465 & 8.8647 & 8.8653 & 8.8655 & 8.8655 \\
10 & 9.0729 & 9.1805 & 9.1820 & 9.1823 & 9.1823 & 9.1823 \\
\hline
\end{tabular}

Table 4.3: The RSNR improvement of MMSE MUD + JVQ decoder through iterations. The CDMA crosscorrelation matrix is $\mathbf{R}_{2}$.

\begin{tabular}{ccccccc}
\hline & \multicolumn{6}{c}{ RSNR (dB) with number of iterations } \\
\cline { 2 - 7 } CSNR (dB) & initial & 1 & 2 & 3 & 4 & 5 \\
\hline 0 & 3.4432 & 3.6953 & 3.7158 & 3.7175 & 3.7176 & 3.7176 \\
2 & 4.5009 & 4.8805 & 4.9179 & 4.9211 & 4.9213 & 4.9214 \\
4 & 5.7974 & 6.2899 & 6.3434 & 6.3479 & 6.3487 & 6.3488 \\
6 & 7.0947 & 7.5698 & 7.6202 & 7.6235 & 7.6250 & 7.6253 \\
8 & 8.1643 & 8.4775 & 8.4965 & 8.4979 & 8.4975 & 8.4971 \\
10 & 8.8671 & 8.9861 & 8.9820 & 8.9793 & 8.9794 & 8.9791 \\
\hline
\end{tabular}




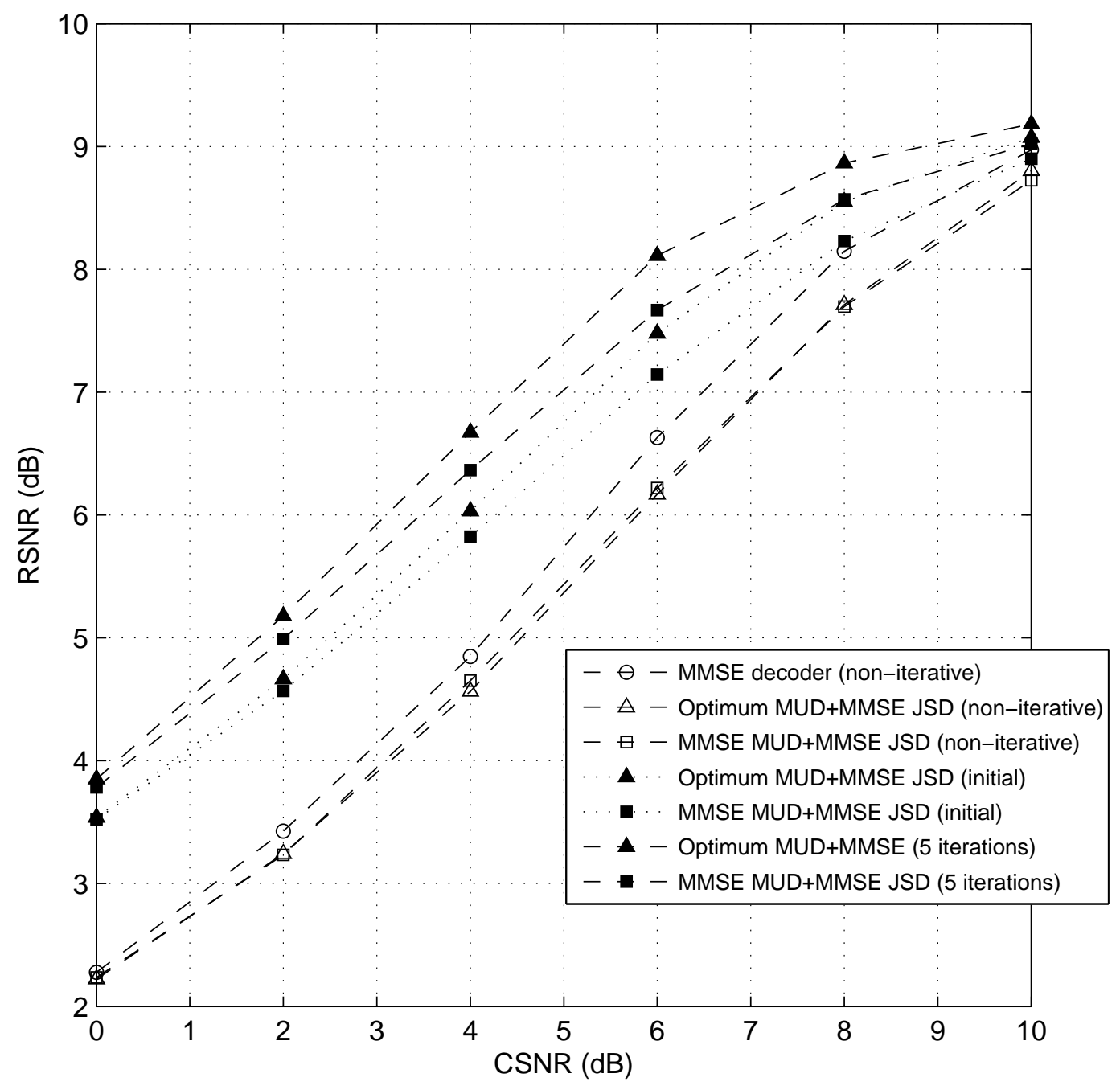

Figure 4.2: Performance comparison between iterative and non-iterative JSC decoding of 4 correlated Gaussian sources over a CDMA channel with destructive interference.

use interleaving interleaving is considerably poor compared to iterative decoders which use interleaving. This is due to destructive interference between highly correlated sources, in this particular CDMA channel. Note that, since the sources are positively correlated, the sensor-pairs which use CDMA signatures with a negative cross-correlation can interfere destructively. On the other hand sensor-pairs with positively correlated signatures can interfere constructively. As can be seen, the iteration between the JVQ decoder and the 
Table 4.4: The RSNR improvement of optimum MUD + JVQ decoder through iterations. The CDMA crosscorrelation matrix is $\mathbf{R}_{2}$.

\begin{tabular}{ccccccc}
\hline & \multicolumn{6}{c}{ RSNR (dB) with number of iterations } \\
\cline { 2 - 7 } CSNR (dB) & initial & 1 & 2 & 3 & 4 & 5 \\
\hline 0 & 3.5497 & 3.8296 & 3.8485 & 3.8508 & 3.8508 & 3.8507 \\
2 & 4.6909 & 5.1397 & 5.1916 & 5.1971 & 5.1966 & 5.1967 \\
4 & 6.0981 & 6.6993 & 6.7734 & 6.7823 & 6.7835 & 6.7838 \\
6 & 7.5102 & 8.0758 & 8.1224 & 8.1253 & 8.1255 & 8.1254 \\
8 & 8.5414 & 8.8504 & 8.8612 & 8.8623 & 8.8623 & 8.8623 \\
10 & 9.0931 & 9.1911 & 9.1939 & 9.1939 & 9.1939 & 9.1939 \\
\hline
\end{tabular}

MUD improves interference cancellation among interleaved channel inputs, and hence the RSNR of the sources (see Table 4.1 and 4.2). For example at a CSNR of $4 \mathrm{~dB}$, for both the optimum MUD and the MMSE-MUD the iterative gain is about $0.5 \mathrm{~dB}$ in RSNR.

Next a CDMA channel with following cross correlation matrix is considered.

$$
\mathbf{R}_{2}=\left(\begin{array}{cccc}
1 & 0.7 & 0.7 & 0.7 \\
0.7 & 1 & 0.7 & 0.7 \\
0.7 & 0.7 & 1 & 0.7 \\
0.7 & 0.7 & 0.7 & 1
\end{array}\right)
$$

For positively correlated sources in this example, this choice of CDMA signatures can expect to give rise to constructive interference. This is confirmed by the simulation results shown in Figure 4.3. In this case, the transmission without interleaving actually outperforms the interleaving-based iterative decoding. The choice of signature waveforms, and hence the cross-correlation matrix is better matched to the inter-source correlation. The superior performance of direct transmission is a manifestation of the fact that, the optimal channel inputs required for transmitting correlated sources over a MAC must also be correlated, see [11]. While the problem of optimizing the channel inputs to achieve minimum MMSE source reconstruction is not addressed in this thesis, this example shows that the optimization of CDMA signatures for correlated sources in conjunction with JSC decoding may offer significant performance gains in MACs. However, notice that the performance 


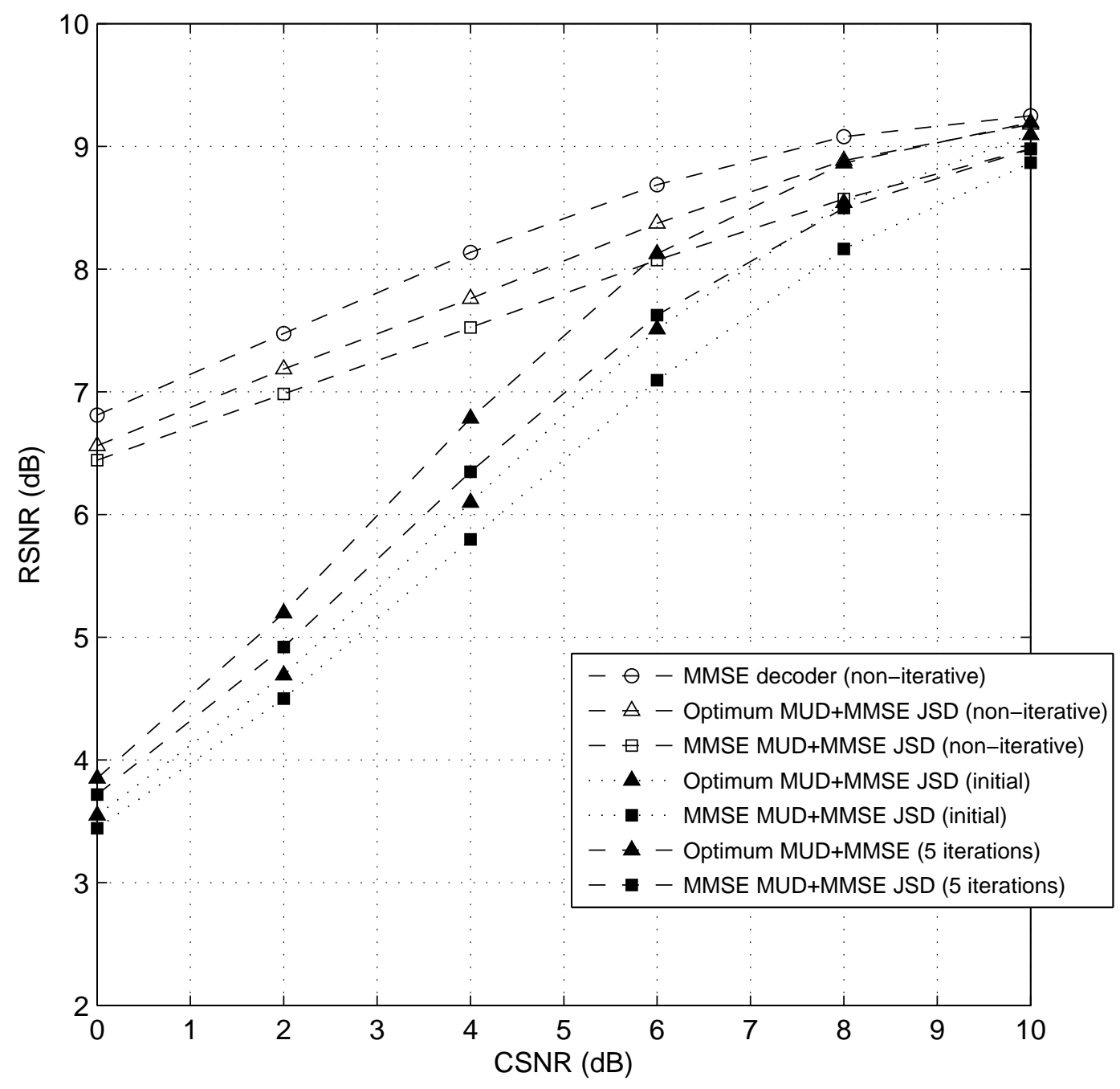

Figure 4.3: Performance comparison between iterative and non-iterative JSC decoding of 4 correlated Gaussian sources over a CDMA channel with constructive interference.

of iterative decoding shown in Figures 4.2 and 4.3 are actually very close despite the differences in the CDMA cross-correlations. This shows that, by rendering the channel inputs independent and then by using the inter-source correlation for interference mitigation in the receiver, the obtained iterative JSC decoders are robust against the effects, destructive or constructive, of particular CDMA signature set in use, compared to non-interleaved transmission. 


\subsection{Near-far resistance of the proposed JSC decoder}

The near-far situation arises in a CDMA system, as the strength of signal received for each user can differ at the receiver. Therefore, depending on the decoding method, the performance of the users may vary with their relative strength. The near-far problem is closely associated with CDMA communication. This is due to the use of non-orthogonal CDMA signatures at the transmitters [5]. In a WSN, there can be many sensors (users) with different transmitter powers and with different proximities to the central receiver, which may lead to a near-far situation. Therefore, the investigation of the performance of the proposed decoder in a near-far situation is also of high interest. A detailed analysis on near-far resistance of MUDs can be found in [34]. However, the analytical results in [34] may not be directly applicable to the JSC decoding considered in this thesis. Therefore the simulation results are obtained to investigate the near-far resistance of the proposed JSC decoder.

In Figure 4.4 and 4.5, the decoder performance in a near-far situation is illustrated. The main focus is on the performance degradation due to the multiple access interference. Therefore the performance is measured in the high CSNR region. In this simulation, User-1 has a constant CSNR of $10 \mathrm{~dB}$, and the interfering users have equal CSNRs and it is varied to obtain the near-far performance of User-1. Two Figures, Figure 4.4 and 4.5 correspond to CDMA cross-correlation matrices $\mathbf{R}_{1}$ and $\mathbf{R}_{2}$ respectively. In both figures, the performance of all investigated JSC decoders remain nearly the same when the interfering users increase their power (i.e. in the $\triangle \mathrm{CSNR}$ region $(0,10) \mathrm{dB}$, where $\left.\triangle \mathrm{CSNR}=\mathrm{CSNR}_{k}-\mathrm{CSNR}_{1}\right)$. With CDMA cross-correlation matrix $\mathbf{R}_{1}$, in Figure 4.4 all the JSC decoders exhibits relatively low performance (i.e. low near-far resistance) when the interfering users decrease their powers (observe the $\triangle \mathrm{CSNR}$ region $(-10,0) \mathrm{dB}$ ). However, it can be observed that iteration of the proposed decoder alleviate the performance drop in the $(-10,0) \mathrm{dB}$ region. This is due to the fact that the extrinsic information about the weak users, delivered by the JVQ decoder are improved by the strong user (User-1), hence the extrinsic information of 


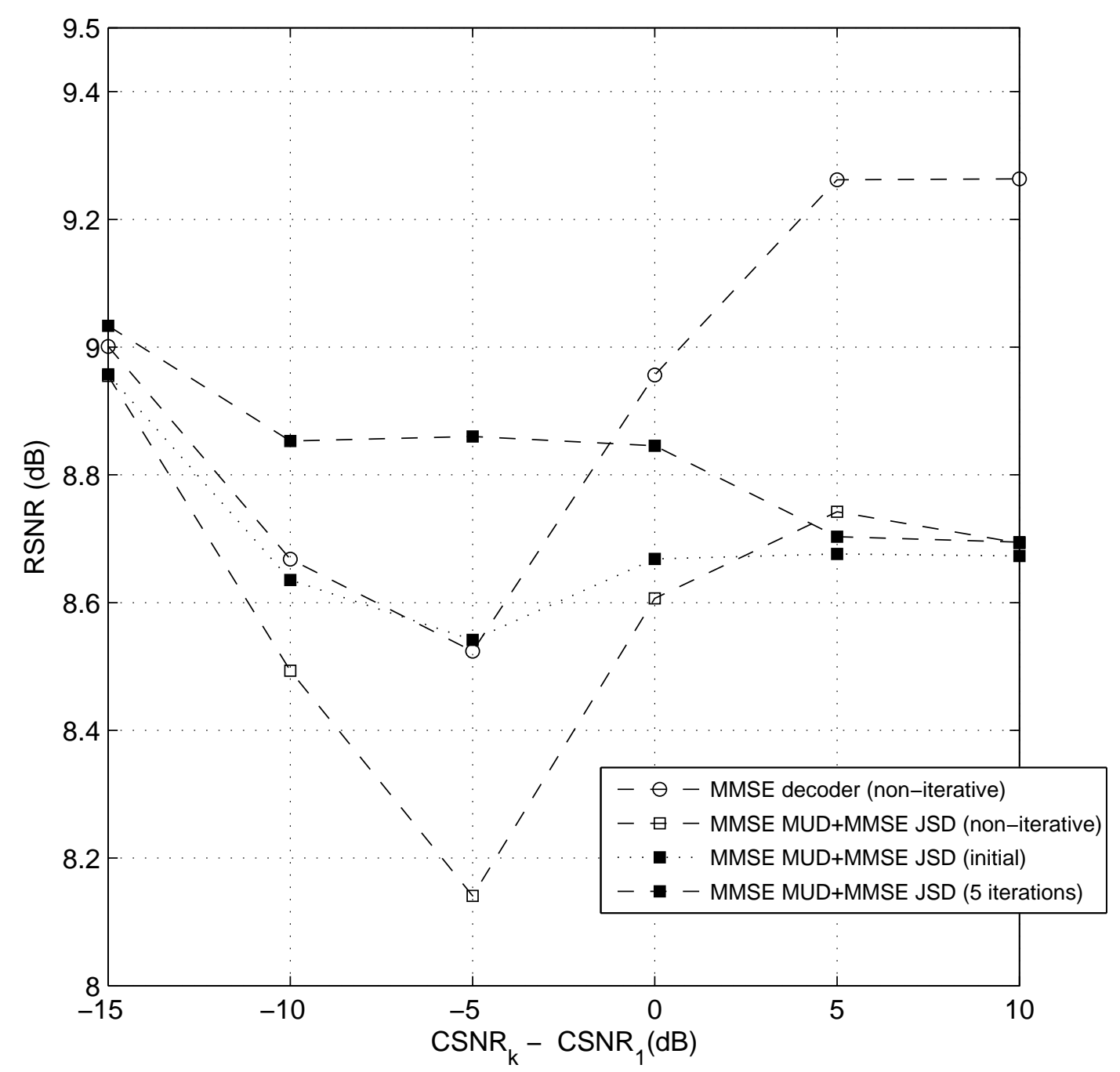

Figure 4.4: Near-far performance (User-1 is illustrated) of the proposed JSC decoder in a four-user system with the CDMA cross-correlation matrix $\mathbf{R}_{1}$. All users employ scalar quantizers. The power of user one is a constant given by $C S N R_{1}=10 \mathrm{~dB}$. The interfering users have equal powers given by $C S N R_{k}-C S N R_{1}$ $(\mathrm{dB}), k=2,3,4$.

User-1 computed at the MMSE MUD is improved. A similar observation can be observed in Figure 4.5. In both the CDMA channels $\mathbf{R}_{1}$ and $\mathbf{R}_{2}$, the proposed iterative JSC decoder exhibits near-far resistance. 


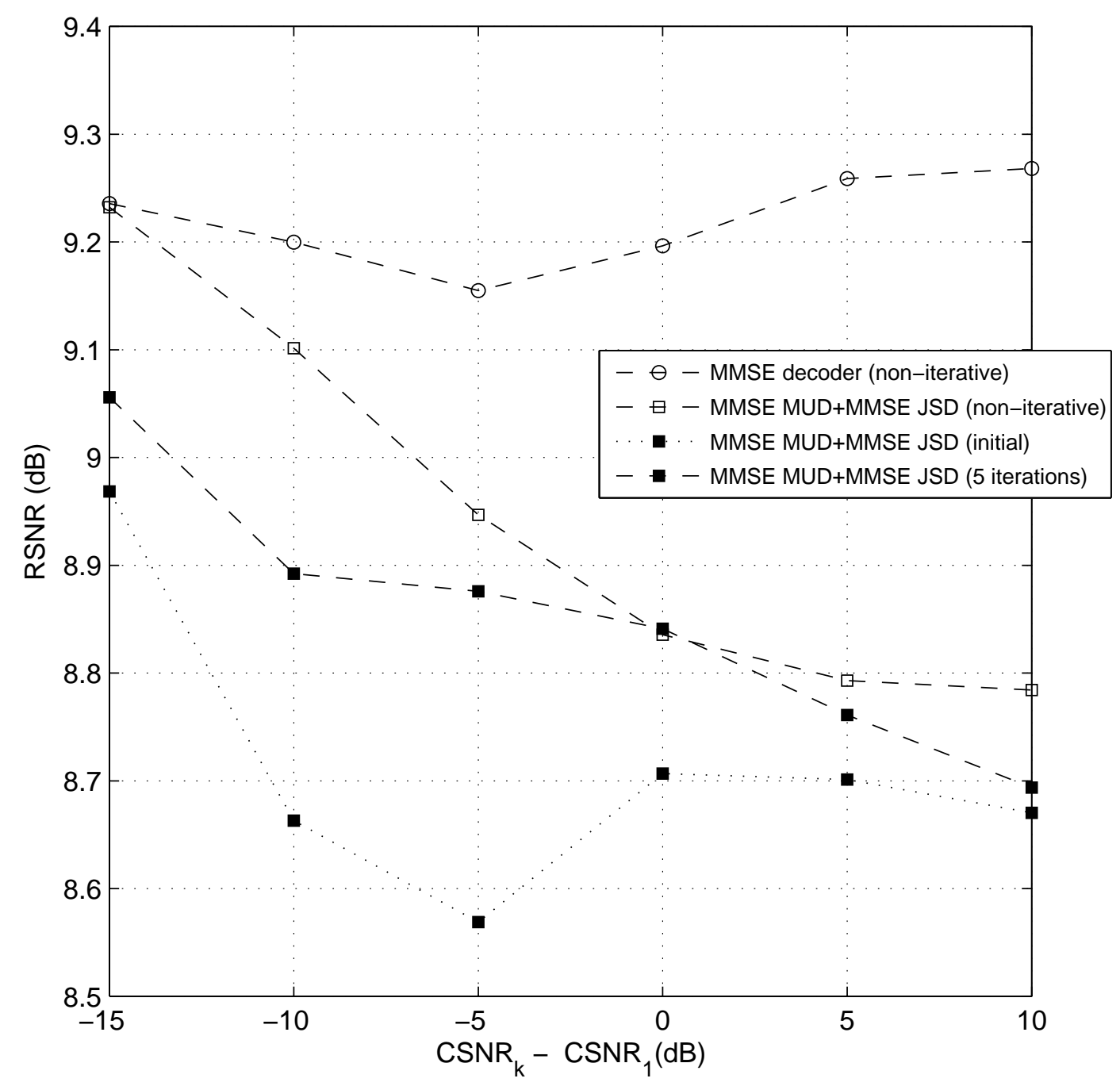

Figure 4.5: Near-far performance of the proposed JSC decoder in a four-user system with the CDMA crosscorrelation matrix $\mathbf{R}_{2}$.

\subsection{Performance comparison of low-complexity JSC de- coders}

Figure 4.6 and 4.7 compares the performance of the proposed decoder with other lowcomplexity JSC decoders. The computational complexity of the JSC decoder proposed in [23] (MMSE MUD + Separate VQ decoders) is $O\left(K^{2}\right)$, which is equal to the computational 


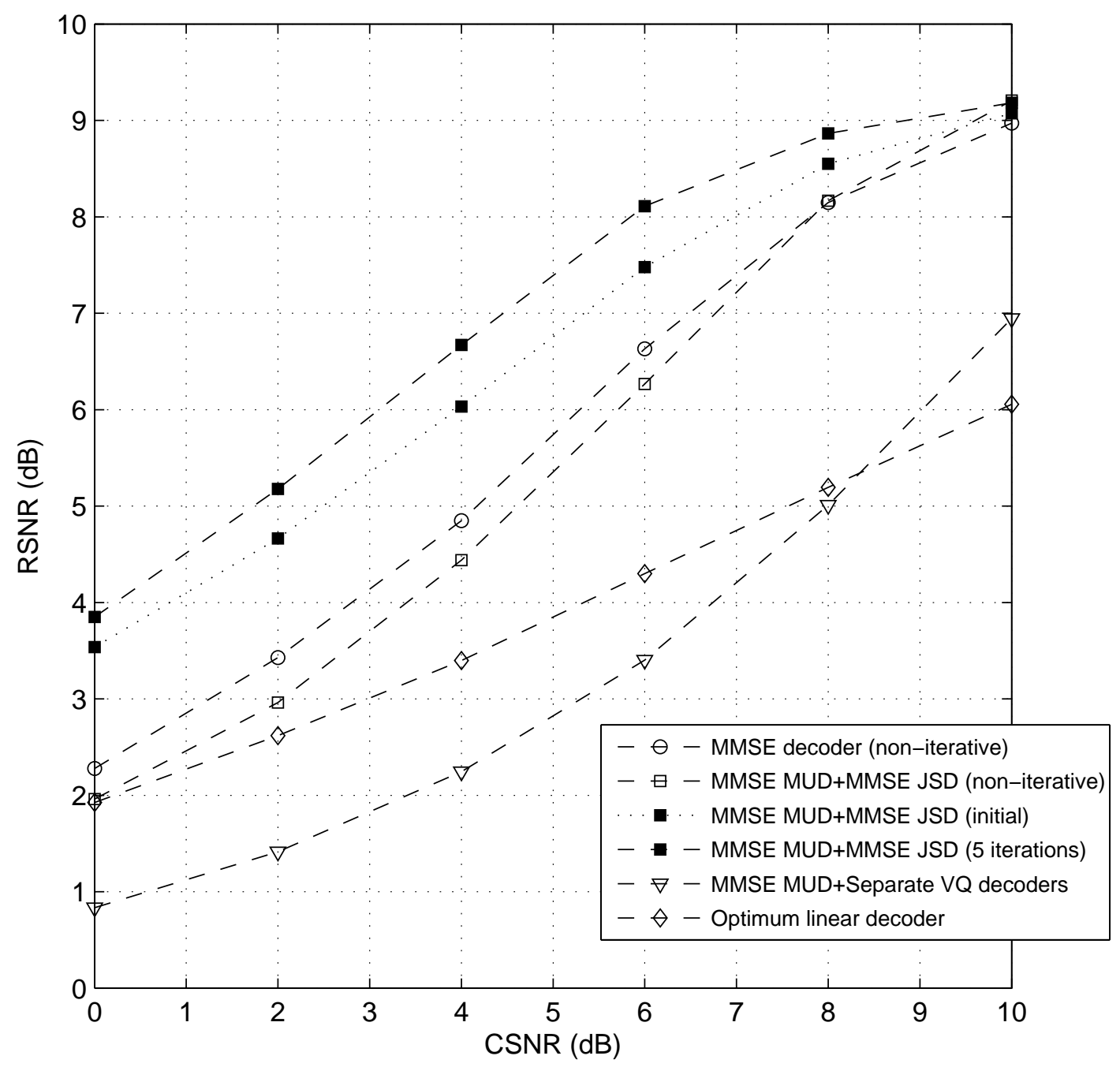

Figure 4.6: A comparison between low-complexity JSC decoders in a four-user system with the CDMA cross-correlation matrix $\mathbf{R}_{1}$. All users employ scalar quantizers.

complexity (for $K>>1$ ) of the proposed decoder in this thesis. And the optimum linear decoder (derived in [35]) has a computational complexity of $O(K)$, which is linear in the number of users in the system.

In Figure 4.7, it can be observed that with the CDMA cross-correlation matrix $\mathbf{R}_{2}$ (i.e constructive channel interferences among the correlated users), the proposed JSC decoder (with iterations) outperforms the JSC decoder in [23] for CSNR $>1.5 \mathrm{~dB}$. This is mainly 


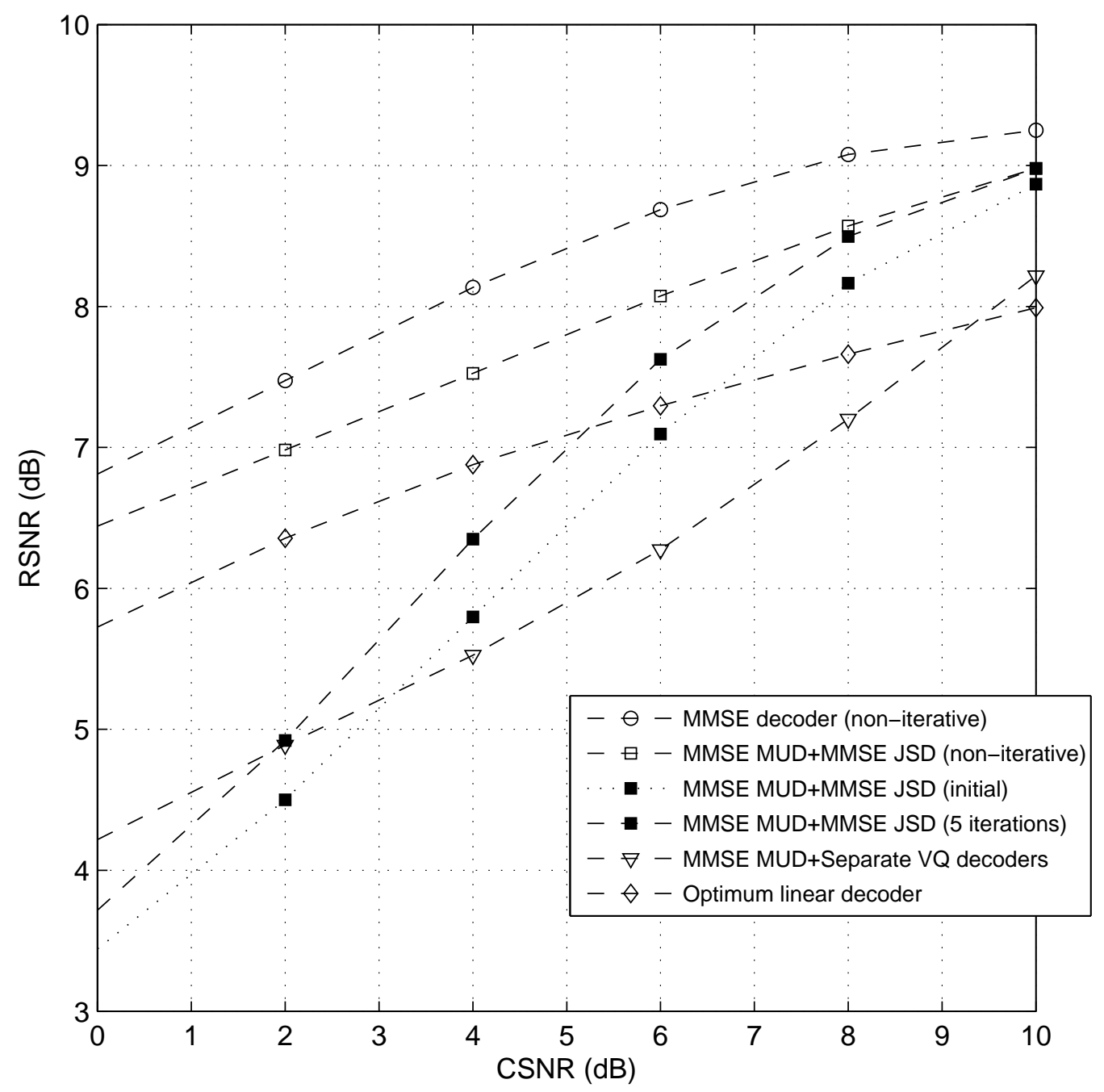

Figure 4.7: A comparison between low-complexity JSC decoders in a four-user system with the CDMA cross-correlation matrix $\mathbf{R}_{2}$.

due to the inability of JSC decoder in [23] to exploit the inter-source correlation at the VQ decoder level. As mentioned earlier in this chapter, the JSC decoder in [23] uses separate individual VQ decoder for each user in tandem with the MMSE-MUD (Note that the the decoder in [23] can be effectively applied in decoding independent sources). The optimum linear decoder exhibits superior performance in the low to medium CSNR region $(0-5) \mathrm{dB}$. In this region, the linear decoder outperforms both the proposed iterative decoder 
and the decoder proposed in [23]. Note that with constructive interference among correlated sources, the linear decoder can exploit the redundancy among users, while in the iterative decoder, MMSE-MUD does not directly account for inter-source correlation, but only through the iterative process. However, it can be observed that, the iterative decoder proposed in this thesis outperforms the optimum-linear decoder in the medium to high CSNR region (0-10) dB. In Figure 4.6, the decoder performance with the CDMA channel $\mathbf{R}_{1}$ (i.e. destructive channel interferences among correlated users) is illustrated. In Figure 4.6, it can be observed that the decoder proposed in this thesis exhibits superior performance compared to the other low-complexity JSC decoders. The gain is about 2-3 dB in the whole CSNR region, compared to the aforementioned two low-complexity decoders. The far inferior performance of the other two JSC decoders is due to the fact that the destructive channel interferences among the correlated users have reduced the information available for decoding. As mentioned in Chapter 3, the proposed iterative decoder avoids this situation by using bit level interleavers.

\subsection{Performance of the proposed decoder on different CDMA channels}

As it was noted in the previous sections, when the data (bits) are directly transmitted (without interleaving) over the CDMA channel, the performance of the decoders highly depend on the CDMA cross-correlation matrix R. To further investigate the relationship between the inter-source correlation and the multi-user interference in the channel, again a foursensor system in a linear-array as shown in Figure 4.1 is considered. The observations are modeled by a mean zero, unit-variance Gaussian random field in which the correlation between any pair of observations $X_{k}$ and $X_{l}$ is given by the power exponential model $([36])$

$$
E\left\{X_{k} X_{l}\right\}=\sigma_{x}^{2} e^{-\left(\Delta d_{k, l}\right)^{2}}
$$




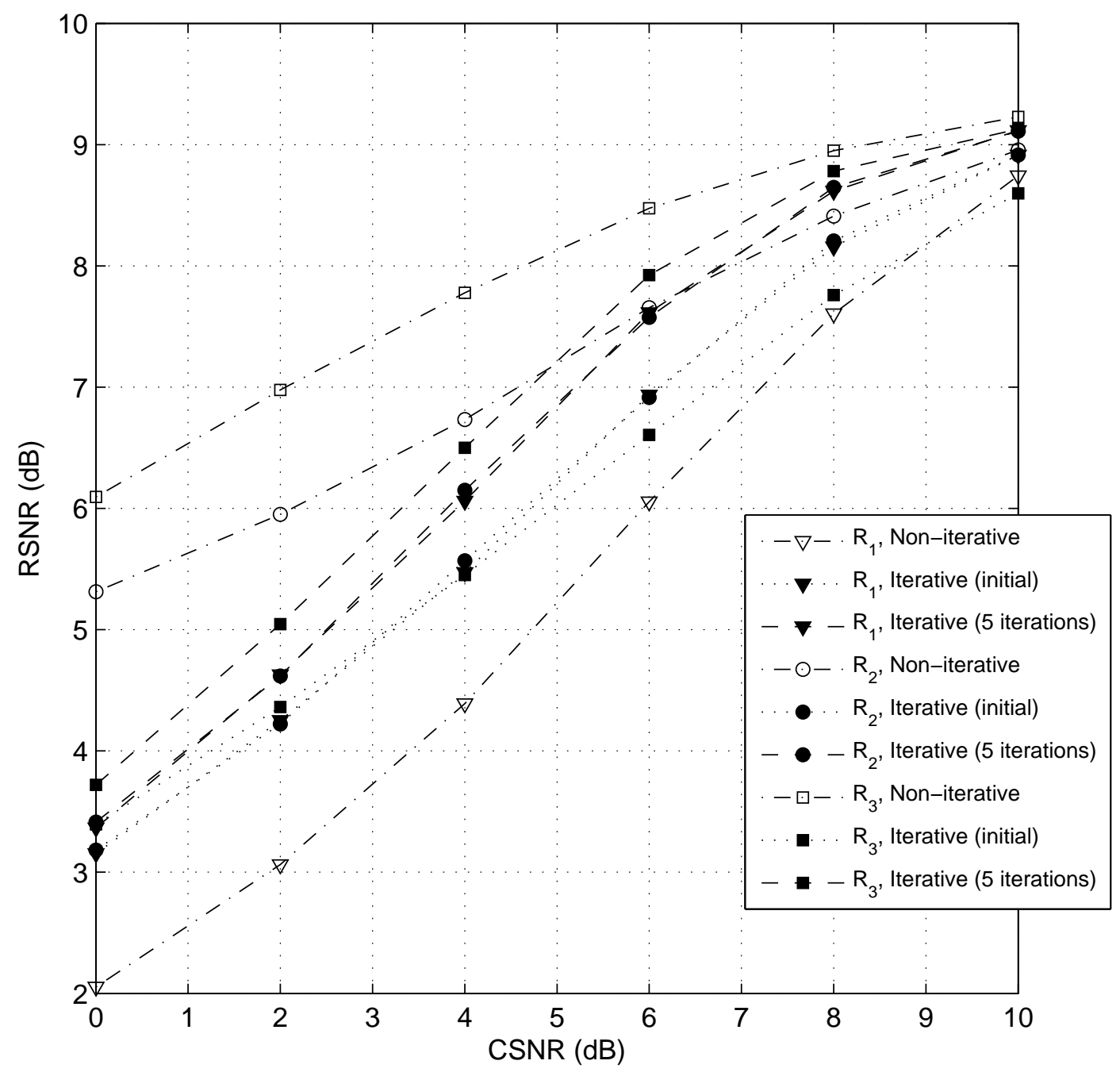

Figure 4.8: Performance of the proposed decoder in three different CDMA channels. The corresponding cross-correlation matrices are given by $\mathbf{R}_{1}, \mathbf{R}_{2}$ and $\mathbf{R}_{3}$.

where $d_{k, l}$ is the distance between the $k$-th and $l$-th sensors and $\Delta$ is a constant. In this simulation distance between two adjacent sensors assumed to be 1.0 , and $\Delta=0.3246$ so that the correlation between two closest sensor observations is 0.9. In this section three CDMA cross-correlation matrices are considered. In addition to the previous two crosscorrelation matrices $\mathbf{R}_{1}$ and $\mathbf{R}_{2}$, a new $\mathbf{R}_{3}$ matrix is also considered.

In Figure 4.8, the performance of both non-iterative (no-interleaving) and iterative (use 
interleaving) decoding of the proposed decoder (MMSE-MUD+MMSE-JSD) is illustrated. The illustrated results with $\mathbf{R}_{1}$ indicates that the use of interleaving and iterative decoding outperforms the non-iterative system with no-interleaving. This is again due to the fact that $\mathbf{R}_{1}$ produces destructive interference between positively correlated users. As can be observed, the results with $\mathbf{R}_{2}$ show that non-iterative decoding outperforms the iterative decoding with interleaving. This is again a confirmation that $\mathbf{R}_{2}$ produces constructive multi-user interference between positively correlated users for the source model given in (4.1). Note that in $\mathbf{R}_{2}$, normalized cross-correlation among all channel signatures are equal (i.e. $\left[\mathbf{R}_{2}\right]_{k, l}=0.7, k \neq l$ ). However, by intuition it is apparent that with no-interleavers, the inter-user interference should follow the level of correlation between the channel inputs, for the better performance. In order to demonstrate this, $\mathbf{R}_{3}$ is chosen such that the crosscorrelation between the CDMA signature waveforms used by any two sensors decays with the distance between the sensors and thus matches the source correlation

$$
\mathbf{R}_{3}=e^{-\left(\Delta_{c} d_{k, l}\right)^{2}}, k \neq l \text { for } k, l=1, \ldots, 4
$$

where $\Delta_{c}=0.3$, so that the cross-correlation between CDMA signatures of the nearest users close to 0.7, which allows us to compare the structure of the $\mathbf{R}_{3}$ with the other two matrices, $\mathbf{R}_{1}$ and $\mathbf{R}_{2}$. The results in Figure 4.8 shows that, when the CDMA channel correspond to $\mathbf{R}_{3}$, decoding with no-interleaving outperforms iterative decoding with interleaving. Both $\mathbf{R}_{2}$ and $\mathbf{R}_{3}$ are able to exploit the inter-source correlation in MAC. Also, it can be observed that the cross-correlation matrix $\mathbf{R}_{3}$ is better matched to the inter-source correlation compared to the cross-correlation matrix $\mathbf{R}_{2}$, for example, with no-interleaving at a CSNR of $4 \mathrm{~dB}$ the gain is about $1 \mathrm{~dB}$ and also with interleaving there is a noticeable gain. These results show that the performance of JSC decoding of correlated sources over no-interleaving is highly dependent on the CDMA cross correlation matrix. While better matched cross-correlation improves the performance of the JSC decoding, a mismatched cross-correlation matrix leads to a performance degradation. On the other hand the proposed iterative decoder with interleaving is more robust against the effects of the CDMA 


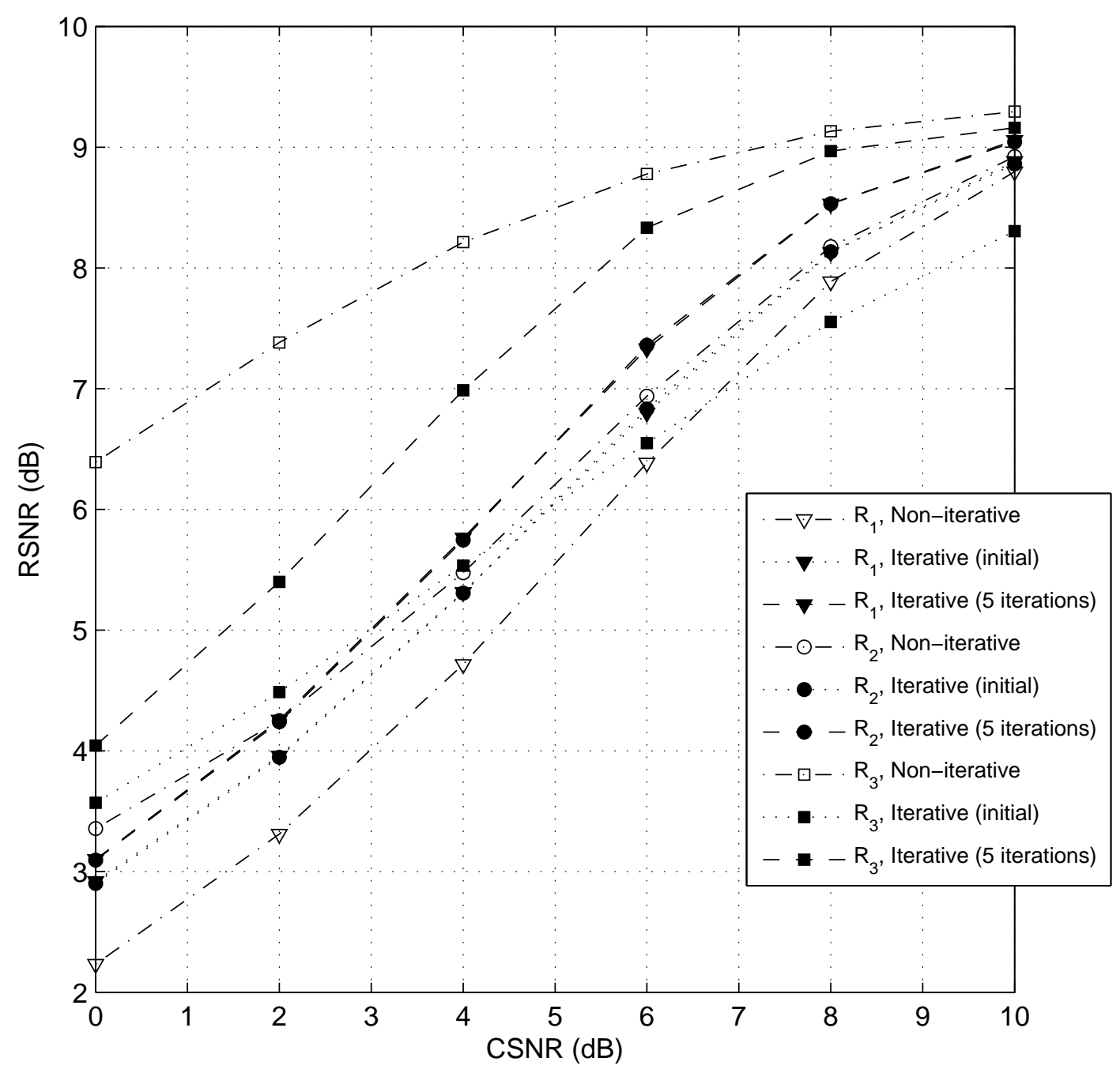

Figure 4.9: Performance of the proposed decoder in an eight user system with different CDMA channels.

cross-correlation matrix. However, determining the optimum set of signatures for correlated sources is not well established in the literature. A theoretical analysis on choosing an optimal set of signature for independent sources appears in [37]. In [20], the effect of choosing a random sequence of CDMA signatures for independent users has been studied.

Figure 4.9 illustrates the performance of the proposed JSC decoder (MMSE-MUD+MMSEJSD) corresponding to three different CDMA cross-correlation matrices, in an eight-user system. The cross-correlation matrices considered have the same structures as previously 
used $\mathbf{R}_{1}, \mathbf{R}_{2}$ and $\mathbf{R}_{3}$ (extended to the eight-user case). The correlated sensor observations are again modeled by the power exponential described earlier. The results in Figure 4.8 and Figure 4.9 shows that the performance curves of the proposed decoder when the CDMA channel corresponds to $\mathbf{R}_{3}$, in both four-user and eight-user systems are very close to each other. However, when the cross-correlation matrix is $\mathbf{R}_{2}$, the performance of the non-interleaved JSC decoding in the eight-user system is considerably low compared to the performance in the four-user system. Notice that in $\mathbf{R}_{3}$ the cross-correlation between the CDMA signatures used by any two users decays exponentially with the distance between two users, which prevents possible destructive interference between uncorrelated sources. However, the cross-correlation between the CDMA signatures in $\mathbf{R}_{2}$ is same between any two users, which can produce destructive interference between uncorrelated users. In Figure 4.9, with non-interleaved decoding is employed, there is considerable performance gain when the corresponding cross-correlation matrix is $\mathbf{R}_{3}$ compared to the cross-correlation matrix $\mathbf{R}_{2}$. For example, at a CSNR of $4 \mathrm{~dB}$ the gain is about $3 \mathrm{~dB}$ in RSNR. However, in the four-user system, the difference between non-interleaved decoding with $\mathbf{R}_{2}$ and $\mathbf{R}_{3}$ is about $1 \mathrm{~dB}$. It is clear that as the number of correlated sources increases, careful optimization of CDMA signatures is even more critical to prevent performance degradation. In the eight-user system also, the robustness of the proposed iterative JSC decoder, against the effects of the CDMA cross-correlation matrix prevails. 


\section{Chapter 5}

\section{Conclusions and Future Work}

Motivated by potential applications in WSNs, in this thesis, we have presented an iterative approach to JSC decoding of multiple correlated sources over a DS-CDMA channel. The prohibitive computational complexity of the MMSE optimal decoding has been addressed by employing a MMSE-MUD and a JVQ decoder in tandem, which iteratively exchange extrinsic information to exploit the correlation among sources. The computational complexity of the JVQ decoder has been controlled by modeling the VQ encoder outputs as a MRF. The proposed iterative decoder has a computational complexity of $O\left(K^{2}\right)(K$ is the number of sources) while the computational complexity of the MMSE optimal decoder is exponential in the number of sources (i.e. $O\left(2^{K}\right)$ ). Therefore the decoder proposed in this thesis is computationally tractable for WSNs with medium to large number of sensors. The performance loss due to the tandem approach adopted here is minimized by extrinsic information passing between the MUD and the JVQ decoder. The iterative decoding is complemented by employing bit-level interleaving, which makes the channel inputs among the users independent. The iterative decoding is useful here due to the large amount of redundancy in the VQ encoder output. The non-iterative (with no-interleavers) version of the aforementioned tandem JSC decoding was also implemented for comparison.

Through simulations, it was found that the iterative gain is considerable regardless of the structure of the CDMA cross-correlation matrix. However, the performance of non- 
iterative decoding is highly dependent on the CDMA cross-correlation matrix. In a system with non-iterative decoding, the multi-user interference among correlated sources could be either constructive or destructive, as no interleavers are employed. Therefore, when the CDMA channel leads to constructive interference among correlated sources the noniterative decoding exhibits superior performance over the iterative decoding. However, the simulation results confirmed that when the non-iterative decoding is employed, the performance degradation due to destructive interference is significant, and even MMSE optimal decoding can show far inferior performance compared to the proposed iterative decoding scheme depending on the assigned CDMA signature set. The simulation results show that iterative decoding is more robust against the effects of the CDMA cross-correlation matrix. The aforementioned robustness of the proposed iterative decoder can be useful in a WSN where the physical location of sensors are unknown. In such WSNs, a given set of CDMA signatures could possibly produce destructive interference among correlated sources. The proposed iterative decoder could minimize possible performance degradations due to destructive channel interference. If in a WSN, the sensor locations are fixed and known, better performance can be achieved by optimizing the CMDA cross-correlation matrix and applying the non-iterative JSC decoding. In this thesis, we have considered such an example where a better matched set of CDMA signatures, for the given source model, been chosen in a heuristic manner. The response of the simulation results in this case indicates the importance of determining the optimal CDMA signature set for correlated sources, a theoretical analysis of this is yet to be done. Through simulations the proposed iterative JSC decoder was also demonstrated to be near-far resistant.

The proposed iterative decoding introduces an additional delay due to the interleavers and the de-interleavers in the system, compared to the non-iterative decoding which does not use interleavers. Therefore, for real time communication, the proposed JSC decoding scheme may not be ideal. The simulation results shows that the convergence of the iterations happens within 4-5 iterations, and therefore the iterative delay is not significant. The robustness against the effect of CDMA cross-correlation matrix has been achieved at the 
expense of an increased communication delay.

\section{Possible future work}

Indications of our simulation results encourage a comprehensive theoretical analysis on the effect of CDMA signature set for correlated sources. And such investigation can be further extended to establish a theoretical method to obtain a near optimal set of signatures for given correlated sources. On the other hand, in a practical set up, for a set of sensors with pre-assigned CDMA signatures, one can conduct research to determine the optimal sensor locations to achieve near optimum decoder performance. The numerical analysis of the near-far resistance problem unveil the potential of the iterative system to provide improved near-far resistance to a stronger user. This in fact gives us some indications that extrinsic information passing between constituent systems can alleviate the dramatic performance drop in a near-far situation. Therefore, an extended analysis on extrinsic information passing in a near-far situation can be interesting. 


\section{Bibliography}

[1] I. Akyildiz, W. Su, Y. Sankarasubramaniam, and E. Cayirci, "A survey on sensor networks," IEEE Communications Magazine, vol. 40, no. 8, pp. 102 - 114, Aug. 2002.

[2] J. Khan, R. Katz, and K. Psiter, "Mobile networking for smart dust," in ACM/IEEE international conference on Mobile computing and networking, Aug. 1999, pp. 271-278.

[3] T. Cover and J. Thomas, Elements of Information Theory, 2nd ed. John Wiley, 2006.

[4] J. Proakis, Digital Communications, 3rd ed. New York: Macgraw Hill, 1995.

[5] S. Verdú, Multiuser Detection, 1st ed. Cambridge University Press, 1998.

[6] S. Pradhan and K. Ramachandran, "Generalized coset codes for distributed binning," IEEE Transactions on Information Theory, vol. 51, pp. 3457-3474, Oct. 2005.

[7] Y. Yang, V. Stankovic, Z. Xiong, and W. Zhao, “On multiterminal source code design," IEEE Transactions on Information Theory, vol. 54, no. 5, pp. 2278-2302, May. 2008.

[8] J. Barros and M. Tuchler, "Scalable decoding on factor trees: A practical solution for wireless sensor networks," IEEE Transactions on Information Theory, vol. 54, no. 2, pp. 284-294, Feb. 2006.

[9] P. Yahampath, "Joint source decoding in large scale sensor networks using Markov random field models," in IEEE International Conference on Acoustics, Speech and Signal Processing, 2009. ICASSP 2009., April 2009, pp. 2769 -2772.

[10] S. Verdú and S. Shamai, "Spectral efficiency of cdma with random spreading," IEEE Transactions on Information Theory, vol. 45, pp. 622-640, Mar. 1999.

[11] S. Ray, M. Medard, M. Effros, and R. Koetter, "On separation for multiple access channels," in Information Theory Workshop, 2006. ITW '06 Chengdu. IEEE, Oct. 2006, pp. 399 -403.

[12] M. Skoglund and P. Hedelin, "Hadamard-based soft decoding for vector quantization over noisy channels," IEEE Transactions on Information Theory, vol. 45, no. 2, pp. 515 -532, Mar. 1999.

[13] V. Vaishampayan and N. Farvardin, "Joint design of block source codes and modulation signal sets," IEEE Transactions on Information Theory, vol. 38, no. 4, pp. 1230 -1248, Jul 1992.

[14] M. Skoglund and P. Hedelin, "Hadamard-based soft decoding for vector quantization over noisy channels," IEEE Transactions on Information Theory, vol. 45, no. 2, pp. 515-532, 1999. 
[15] C. E. Shannon, "A mathematical theory of communication," Bell Syst. Tech. J., vol. 27, pp. 379-423, 623-656, July and Oct. 1948.

[16] C. Shannon, "Communication in the presence of noise," Proceedings of the IRE, vol. 37, no. 1, pp. 10 - 21, Jan. 1949.

[17] A. Gresho and R. M. Gray, Vector Quantization and Signal Compression. Kluwer Academic Publishers, 1992.

[18] S. Lloyd, "Least squares quantization in PCM," IEEE Transactions on Information Theory, vol. 28, no. 2, pp. 129 - 137, Mar. 1982.

[19] H. Landau and H. Pollak, "Fourier analysis and uncertainty-iii: The dimension of the space of essentially time- and band-limited signals," Bell Technical Journal, vol. 41, pp. 1295-1336, 1962.

[20] A. Grant and P. Alexander, "Random sequence multisets for synchronous code-division multiple-access channels," IEEE Transactions on Information Theory, vol. 44, no. 7, pp. 2832 -2836, Nov. 1998.

[21] M. Skoglund and T. Ottosson, "Soft multiuser decoding for vector quantization over a CDMA channel," IEEE Transactions on Communications, vol. 46, no. 3, pp. 327 -337, Mar. 1998.

[22] C. Illangakoon and P. Yahampath, "On joint source-channel decoding and interference cancellation in CDMA-based large-scale wireless sensor networks," IEEE International Conference on Acoustics, Speech and Signal Processing, 2013. ICASSP 2013.

[23] H. Nguyen, "A soft decoding scheme for vector quantization over a CDMA channel," IEEE Transactions on Communications, vol. 53, no. 10, pp. 1603 - 1608, Oct. 2005.

[24] H. Poor, An Introduction to Signal Detection and Estimation, 2nd ed. New York: SpringerVerlag, 1994.

[25] T. Cover, A. Gamal, and M. Salehi, "Multiple access channels with arbitrarily correlated sources," IEEE Transactions on Information Theory, vol. 26, no. 6, pp. 648 - 657, Nov. 1980.

[26] X. Wang and H. Poor, "Iterative (turbo) soft interference cancellation and decoding for coded CDMA," IEEE Transactions on Communications, vol. 47, no. 7, pp. 1046 -1061, Jul. 1999.

[27] J. Hagenauer, "Forward error correcting for CDMA systems," in IEEE 4th International Symposium on Spread Spectrum Techniques and Applications Proceedings, vol. 2, Sep. 1996, pp. $566-569$.

[28] H. El Gamal and E. Geraniotis, "Iterative multiuser detection for coded CDMA signals in AWGN and fading channels," IEEE Journal on Selected Areas in Communications, vol. 18, no. 1, pp. 30-41, Jan. 2000.

[29] J. Hagenauer and N. Gortz, "The turbo principle in joint source-channel coding," in IEEE proceedings, Information Theory Workshop, 2003. Proceedings. 2003 IEEE, Apr. 2003, pp. $275-278$.

[30] N. Gortz, "On the iterative approximation of optimal joint source-channel decoding," IEEE Journal on Selected Areas in Communications, vol. 19, no. 9, pp. 1662 -1670, Sep. 2001. 
[31] H. Poor and S. Verdú, "Probability of error in mmse multiuser detection," IEEE Transactions on Information Theory, vol. 43, no. 3, pp. 858 -871, May 1997.

[32] F. Kschischang, B. Frey, and H.-A. Loeliger, "Factor graphs and the sum-product algorithm," IEEE Transactions on Information Theory, vol. 47, no. 2, pp. 498-519, Feb. 2001.

[33] R. Yasaratna and P. Yahampath, "Design of scalable decoders for sensor networks via Bayesin network learning," IEEE Transaction on Communication, vol. 57, no. 10, pp. 2868-2871, Oct. 2009.

[34] R. Lupas and S. Verdú, "Near-far resistance of multiuser detectors in asynchronous channels," IEEE Transactions on Communications, vol. 38, no. 4, pp. 496 -508, Apr. 1990.

[35] H. Nguyen and W. Fernando, "Optimum linear decoding of vector quantization transmitted over a CDMA channel,” IET Communications, vol. 1, no. 6, pp. 1206 -1211, Dec. 2007.

[36] M. Vuran, O. Akan, and I. Akylidz, "Spatio-temporal correlation: theory and applications for wireless networks," Computer Networks, vol. 45, p. 245259, 2004.

[37] M. Rupf and J. Massey, "Optimum sequence multisets for synchronous code-division multiple-access channels," IEEE Transactions on Information Theory, vol. 40, no. 4, pp. 12611266, Jul. 1994. 